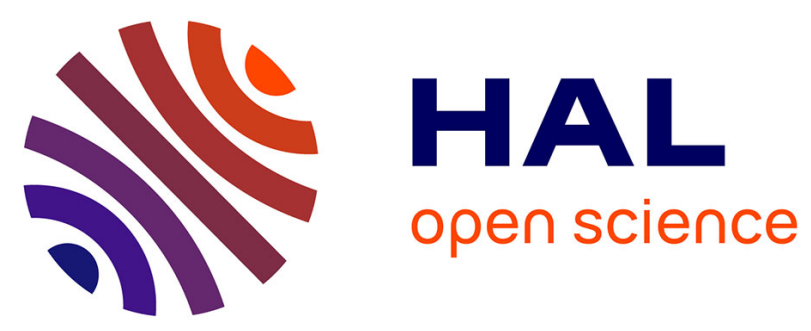

\title{
Les programmes aéroportés Mozaic et Iagos (1994-2008)
}

Jean-Pierre Cammas, Gilles Athier, Damien Boulanger, Françoise Chéroux, Jean-Marc Cousin, Françoise Girod, Fernand Karcher, Kathy S. Law, Philippe Nédélec, Vincent-Henri Peuch, et al.

\section{- To cite this version:}

Jean-Pierre Cammas, Gilles Athier, Damien Boulanger, Françoise Chéroux, Jean-Marc Cousin, et al.. Les programmes aéroportés Mozaic et Iagos (1994-2008). La Météorologie, 2008, 62, pp.PP.18-31. meteo-00350096

\section{HAL Id: meteo-00350096}

\section{https://hal-meteofrance.archives-ouvertes.fr/meteo-00350096}

Submitted on 4 Jun 2021

HAL is a multi-disciplinary open access archive for the deposit and dissemination of scientific research documents, whether they are published or not. The documents may come from teaching and research institutions in France or abroad, or from public or private research centers.
L'archive ouverte pluridisciplinaire $\mathbf{H A L}$, est destinée au dépôt et à la diffusion de documents scientifiques de niveau recherche, publiés ou non, émanant des établissements d'enseignement et de recherche français ou étrangers, des laboratoires publics ou privés. 


\title{
Les programmes aéroportés Mozaic et lagos (1994-2008)
}

\section{Résumé}

Mozaic est un programme initié en 1993 par des scientifiques européens, Airbus et des compagnies aériennes, pour mesurer les concentrations d'ozone, de vapeur d'eau, de monoxyde de carbone et des oxydes d'azote, en utilisant cinq avions longcourrier en service commercial. La base de donnée de Mozaic, unique en son genre, permet d'étudier les processus physiques et chimiques atmosphériques, pour mieux comprendre les problèmes qui se posent à l'échelle globale sur le climat et la qualité de l'air. Iagos prépare les bases d'une infrastructure distribuée pour l'observation globale de la composition chimique, des aérosols, des nuages et des traînées de condensation à partir d'avions commerciaux en service. Pour cet objectif, de nouveaux instruments sont développés sur la base des instruments Mozaic.

\section{Abstract}

Mozaic and lagos (1994-2008): measuring reactive gases in the atmosphere with airliners

Mozaic is a program initiated in 1993 by European scientists, Airbus and airlines, to collect experimental data on ozone, water vapour, carbon monoxide and nitrogen oxides, by five long range passenger airliners in commercial use. A large and unparalleled database of measurements is being built to allow studies of chemical and physical processes in the atmosphere, and hence to better understand their effects on global climate and air quality. Iagos is preparing a distributed infrastructure for observations of atmospheric composition, aerosols, clouds and contrails on the global scale from commercial in-service aircraft. For this purpose, new instrument packages are being developed based on the former Mozaic instrumentation.

\author{
Jean-Pierre Cammas ${ }^{(1)}$, Gilles Athier ${ }^{(1)}$, Damien Boulanger ${ }^{(1)}$, \\ Françoise Chéroux ${ }^{(2)}$, Jean-Marc Cousin ${ }^{(1)}$, Françoise Girod ${ }^{(5)}$, \\ Fernand Karcher ${ }^{(2)}$, Kathy Law ${ }^{(4)}$, Philippe Nédélec ${ }^{(1)}$, \\ Vincent-Henri Peuch ${ }^{(2)}$, Herman Smit ${ }^{(3)}$, Magali Stoll ${ }^{(2)}$, \\ Delphine Texier ${ }^{(6)}$, Valérie Thouret ${ }^{(1)}$, Andreas Volz-Thomas ${ }^{(3)}$ \\ et Régina Zbinden ${ }^{(1)}$. \\ (1) Laboratoire d'aérologie, CNRS - Université de Toulouse (UMR 5560), \\ Observatoire Midi-Pyrénées - France \\ (2) Centre national de recherche météorologique (CNRM) \\ Météo-France - Toulouse - France \\ (3) Institut für Chemie und Dynamik der Geosphäre 2 \\ Forschungszentrum Jülich - Allemagne \\ (4) Service d'aéronomie - Université Paris 6 - Paris - France \\ (5) Centre national d'études spatiales - Toulouse - France \\ (6) Observatoire Midi-Pyrénées - UMS 831 - Toulouse - France
}

'ozone et la vapeur d'eau sont des constituants clés de l'atmosphère terrestre car ils jouent un rôle déterminant dans le couplage entre la chimie atmosphérique et le climat. Toute perturbation significative de leur distribution impacte notre climat. La vapeur d'eau est le gaz à effet de serre le plus important et ses changements de phase participent à la thermodynamique de l'atmosphère. L'ozone est aussi un gaz à effet de serre ; le potentiel de forçage radiatif de ses perturbations dans la haute troposphère est aussi fort que celui du gaz carbonique. Tous deux précurseurs des radicaux hydroxyles, l'ozone et la vapeur d'eau ont un rôle majeur dans la chimie de la troposphère, en étant fortement impliqués dans le cycle de vie de plusieurs autres gaz trace naturels et anthropiques. Dans la couche limite planétaire, la production d'ozone, par la réaction entre ses précurseurs issus de la pollution atmosphérique (oxydes d'azote $\mathrm{NO}_{\mathrm{x}}$, hydrocarbures imbrûlés comme le monoxyde de carbone $\mathrm{CO}$, composés organiques volatiles...) et l'oxygène de l'air en présence du rayonnement solaire, peut entraîner des problèmes de santé chez les personnes fragiles et des diminutions de croissance de nos récoltes. La multitude des échelles de temps et d'espace impliquées par la photochimie et le transport de l'ozone fait que l'ozone troposphérique a une énorme variabilité, qui rend très complexe l'analyse de son bilan par lequel, cependant, passe la compréhension de ses variations interannuelles, de ses tendances et de son impact sur le climat.

Notre connaissance actuelle du bilan de l'ozone troposphérique, délivrée par les modèles (globaux de chimie-transport, climatiques régionaux et régionaux pour la qualité de l'air), est encore très insuffisante et révèle de trop grandes incertitudes sur les interactions entre processus de transport et de chimie. Elle est encore largement entretenue par les déficiences des réseaux d'observations. Au sol, les besoins d'observation dans les milieux urbains et ruraux sont relativement bien couverts par différents réseaux d'observations : agences de qualité de l'air, stations des réseaux nationaux [en France, Emera pour les sites ruraux, PAES pour les stations de montagne] (voir Gheusi et al., 2008) et internationaux (GAW et Emep). Les besoins d'observation les plus cruciaux sont sur les profils verticaux dans la troposphère et la stratosphère. Au niveau international, les sondages ozone par ballon sont trop 
peu fréquents (hebdomadaires au mieux) et avec une couverture géographique très insuffisante. Les capacités actuelles de télédétection satellite en chimie atmosphérique concernent essentiellement la stratosphère. Pour la troposphère, elle donnent accès à des colonnes troposphériques encore entachées de fortes incertitudes, ce qui rend difficile leur assimilation ou leur rôle de variables de contrôle pour les modèles. Les applications donnant accès à de l'information objective sur le profil troposphérique ne font que commencer (par exemple, Mopitt pour le CO, ACE et Iasi pour l' $\mathrm{O}_{3}$ et le $\mathrm{CO} \ldots$ ). Les besoins de valider ces techniques spatiales par des mesures in situ précises existeront toujours. Les campagnes de mesures fournissent des jeux de données généralement assez complets, cependant biaisés par une focalisation sur un objectif précis de recherche et un manque de représentativité aux échelles plus larges que la région et à l'échelle climatique.

L'idée d'équiper des avions commerciaux avec une instrumentation automatique pour pallier les insuffisances du réseau d'observations globales de la chimie atmosphérique est apparue dans les années 1970. De par les caractéristiques des vols des avions commerciaux, l'objectif de ces réseaux d'observations est d'avoir des mesures fréquentes (profils troposphériques et phases de croisière entre haute troposphère et basse stratosphère), sur une couverture géographique très large, avec un coût de revient très compétitif dans la mesure où les compagnies aériennes peuvent offrir le coût de transport de l'instrumentation.
La première tentative a été faite par la Nasa avec le programme Gasp (Global Atmospheric Sampling Programme, 1975-1979) dans lequel un Boeing 747 a été instrumenté avec des mesures d'ozone (Nastrom, 1979). L'idée a ensuite été réactivée dans les années 1990 avec plusieurs projets de recherche européens et japonais. Mozaic [Measurements of Ozone, Water Vapour, Carbon Monoxyde and Nitrogen Oxydes by Airbus inservice aircraft ; http://mozaic.aero.obsmip.fr/web/] (voir Marenco et al., 1998), a démarré en 1993 comme un projet de recherche soutenu par Airbus, le Centre national de la recherche scientifique (CNRS), Météo-France, l'université de Cambridge, la Commission européenne et les compagnies aériennes Lufthansa, Air France, Austrian et Sabena. Mozaic est encore opérationnel en 2008. Noxar [Nitrogen Oxides and Ozone along Air Routes] (voir Brunner et al., 2001), projet de l'Institut fédéral de technologie suisse soutenu par la Commission européenne et la compagnie aérienne Swiss, a été opérationnel de 1995 à 1997. Caribic [Civil Aircraft for the Regular Investigation of the Atmosphere Based on an Instrument Container] (voir Brenninkmeijer et al., 2007), a été initié en 1994 comme un projet de recherche soutenu par l'Institut Max-Planck, la Commission européenne et la compagnie Lufthansa. Caribic est encore opérationnel en 2008 et fait maintenant partie du consortium Iagos (In-service Aircraft for a Global Observing System ; http://www.fz-juelich.de/icg/icg2/iagos). Contrail (Comprehensive Observation Network for Trace Gases by Airliner) est un projet soutenu depuis 1993 par l'Institut national des études

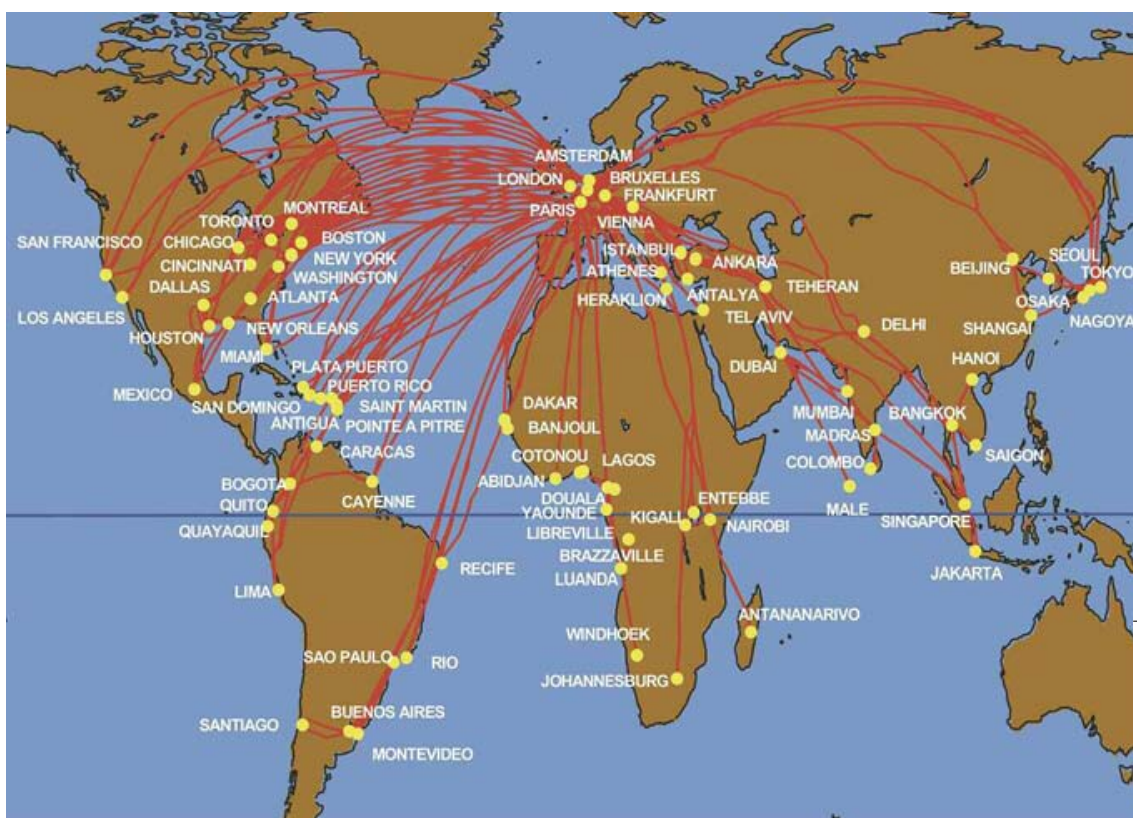

environnementales à Tsukuba (Japon) et par la fondation de la compagnie Japan Airlines.

L'objectif de cet article est de présenter les programmes Mozaic et Iagos. L'article décrit l'instrumentation embarquée et les principaux résultats acquis. Les perspectives de transformation du projet de recherche Mozaic en une infrastructure de recherche européenne, Iagos-ERI (In-service Aircraft for a Global Observing System - European Research Infrastructure), sont ensuite développées.

\section{Le programme Mozaic}

Les objectifs principaux de ce programme étaient de fournir des observations de vapeur d'eau, d'ozone et de ses précurseurs dans les zones très mal observées de l'atmosphère, en particulier la zone HTBS (Haute troposphère basse stratosphère), et d'évaluer l'impact de la flotte aérienne commerciale subsonique sur l'atmosphère, notamment pour l'ozone. Trois phases ont pris place entre 1993 et 2004 sous le cofinancement de la Commission européenne. Les mesures ont commencé en 1994, avec l'installation des instruments pour la mesure de l'ozone $\left(\mathrm{O}_{3}\right)$ et de l'humidité relative $\left(\mathrm{H}_{2} \mathrm{O}\right)$ à bord de cinq avions commerciaux. En 2001, les avions ont été équipés d'instruments pour la mesure du monoxyde de carbone $(\mathrm{CO})$. Un des cinq avions a été équipé pour la mesure des oxydes d'azote $\mathrm{NO}_{\text {y }}$ (regroupement de tous les composés azotés actifs). Depuis 2004 , le programme continue avec des supports nationaux de l'Institut national des sciences de l'univers CNRS (Insu-CNRS), de l'Observatoire Midi-Pyrénées, de Météo-France et du FZJ (Forschungszentrum Jülich, Allemagne). Actuellement, trois des cinq avions Mozaic initiaux sont toujours en service (deux de Lufthansa et un d'Air Namibia). Plus de 28000 vols ont été effectués depuis 1994 avec la couverture géographique indiquée sur la figure 1.
Figure 1 - Carte de la couverture géographique des vols Mozaic. Chacun de ces vols comprend deux profils verticaux entre le sol et 9 à $12 \mathrm{~km}$ d'altitude, avec environ huit heures de données dans la zone haute troposphère - basse stratosphère. 
Mozaic a fourni des informations originales sur les distributions de $\mathrm{H}_{2} \mathrm{O}, \mathrm{O}_{3}$, $\mathrm{CO}$ et $\mathrm{NO}_{\mathrm{y}}$ dans la zone HTBS et sur la structure verticale de ces espèces dans la troposphère. Les données sont exploitées par les principaux investigateurs de Mozaic et les coïnvestigateurs répartis dans plusieurs instituts de recherche internationaux, résultant en treize thèses et plus de cent vingt publications dans des journaux à comité de lecture (voir sur http://mozaic.aero.obsmip.fr/web/). Les thèmes de recherche incluent les variations saisonnières, géographiques et interannuelles des gaz en trace en relation avec leurs sources et la dynamique de l'atmosphère, l'évaluation des modèles globaux de chimie-transport et l'évaluation des techniques d'inversion pour les produits de chimie atmosphériques à partir d'observations spatiales.

\section{Instrumentation Mozaic et opération}

L'ozone est mesuré par absorption UV (Thermo Instruments, Model 49-103). Les instruments sont étalonnés avant et après chaque période de déploiement (environ tous les douze mois). Des contrôles de qualité sur les biais et sur les facteurs de l'étalonnage sont effectués en vol avec un générateur interne d'ozone et par comparaison des mesures pour les avions volant en quasicoïncidence spatiotemporelle. La précision est estimée à $\pm[2 \mathrm{ppbv}+2 \%]$.

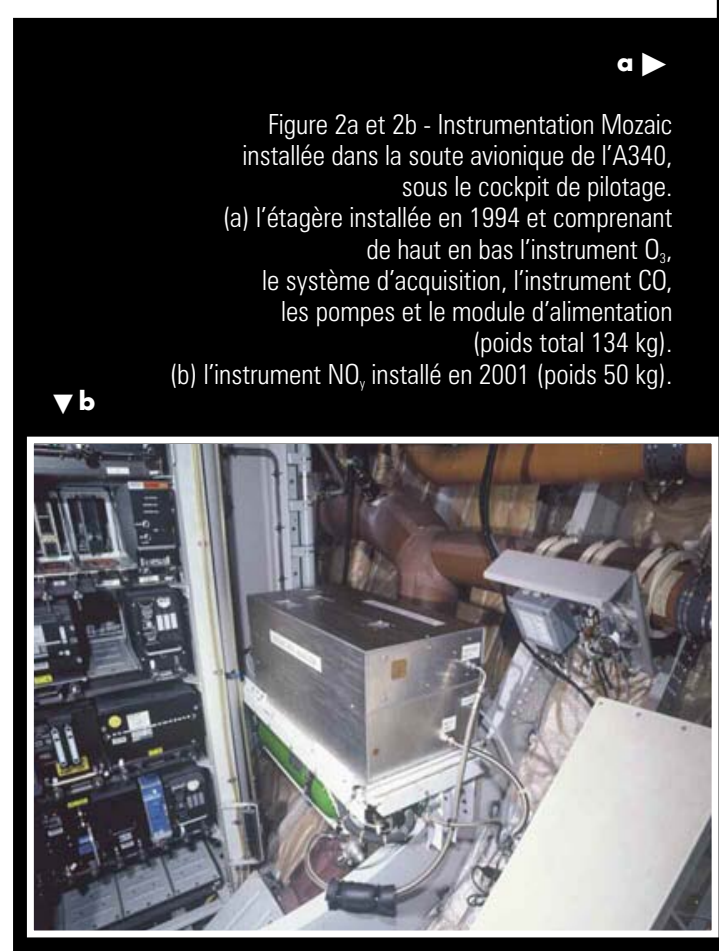

Les procédures de contrôle de qualité sont restées invariables au cours de Mozaic de façon à éviter des artefacts instrumentaux dans les séries temporelles (Thouret et al., 1998b). Une comparaison des deux premières années des données Mozaic avec les données du réseau international des sondages ozone par ballon a montré un bon accord (Thouret et al., 1998b).

Pour la mesure de l'humidité relative et de la température, un minicapteur aéroporté à thermistance est utilisé (Vaisala, Humicap-H et Pt-100). Les minicapteurs sont intégrés suivant le standard aéronautique (TAT) et sont étalonnés avant et après déploiement (environ tous les cinq cents vols) dans une chambre climatique simulant les conditions de vol. De ces étalonnages résulte une incertitude à $2 \sigma$ de $\pm(5-10) \%$ pour l'humidité relative et de $\pm(0,5-0,7) \mathrm{K}$ pour la température (Helten et al., 1998). Les performances en vol ont été confirmées par comparaisons avec des instruments de référence au cours de missions aéroportées dédiées (Helten et al., 1999). Le temps de réponse du capteur d'humidité est d'environ une à deux minutes suivant la température.

Pour la mesure du CO, la technique de corrélation par filtre IR est employée (Thermo Environmental Instruments, Model 48CTL). Bien que moins sensi-

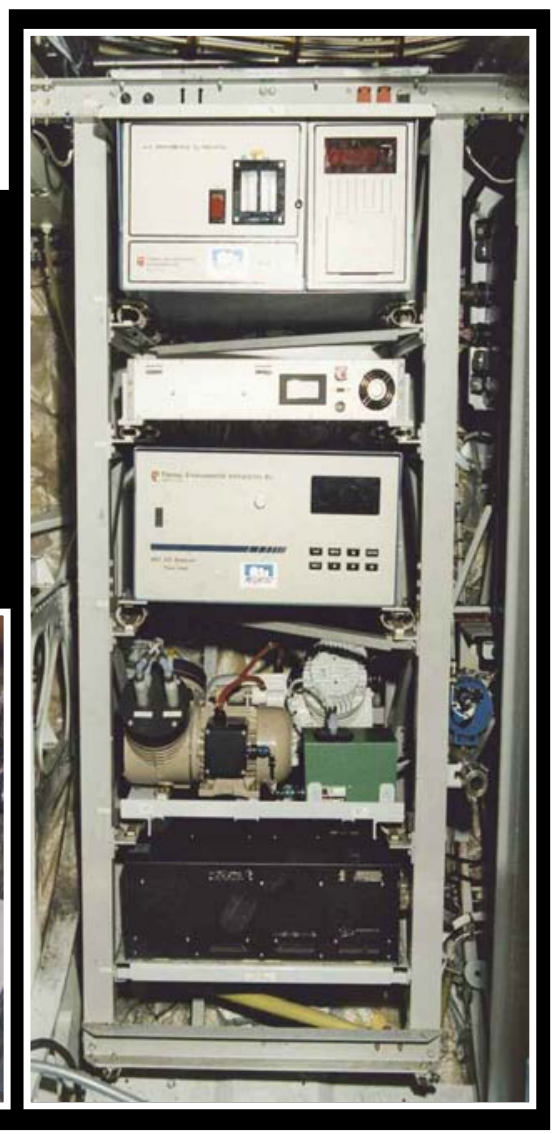

ble que d'autres instruments (diodes laser accordées ou résonance fluorescente), l'instrument IR est bien moins complexe et fournit une excellente stabilité, ce qui est crucial pour une opération continue sans maintenances rapprochées. La sensibilité de l'instrument (normalement 10 ppbv pour $300 \mathrm{~s}$ d'intégration) a été améliorée grâce à plusieurs modifications (Nédélec et al., 2003), incluant des mesures de zéro périodiques, l'utilisation d'un nouveau détecteur IR à température régulée et l'augmentation de la pression dans la cellule d'absorption. La précision atteinte avec cet instrument modifié est de \pm 5 ppbv ou de $\pm 5 \%$ pour un temps de réponse de $30 \mathrm{~s}$.

$\mathrm{NO}_{\mathrm{y}}$ (la somme de $\mathrm{NO}$ et de ses produits d'oxydation atmosphérique) est mesuré par chemiluminescence de NO avec $\mathrm{O}_{3}$ après une réduction catalytique des différents composés de $\mathrm{NO}_{\mathrm{y}}$ en $\mathrm{NO}$ par déposition de traces d'hydrogène sur une surface en or chauffée (VolzThomas et al., 2005). L'instrument a été spécialement conçu pour une opération en continu dans un avion commercial et se caractérise par un poids de $50 \mathrm{~kg}$, un fonctionnement sans surveillance de cinq semaines au minimum et un autoétalonnage en vol. La sensibilité de 0,4-0,7 cps/ppt permet une limite de détection statistique inférieure à $\pm 50 \mathrm{ppt}$ pour un temps d'intégration de 4 s et de $\pm 150-300$ ppt au maximum de résolution de l'instrument à $10 \mathrm{~Hz}$. Une comparaison en vol avec un instrument de recherche opéré par l'ETH-Zurich (Pätz et al., 2006) a donné de bons résultats, en accord avec l'incertitude combinée des deux instruments $( \pm 7 \%)$.

Tous ces instruments sont montés sur une étagère 19 pouces $(134 \mathrm{~kg})$ dans le compartiment avionique, sous le poste de pilotage (figure 2), avec les tubes d'admission des gaz montés sur le fuselage à environ 7 mètres du nez de l'avion. Le système est automatique et ne demande aucune intervention de l'équipage. Les compagnies aériennes (figure 3) transportent gratuitement l'instrumentation et effectuent une maintenance minimale, c'est-à-dire vérification des fonctionnalités, remplacement des disquettes d'archivage et du capteur d'humidité. Lufthansa opère deux avions depuis 1994. Air France et Austrian ont opéré un avion chacun de 1994 à 2005. Après l'arrêt de Sabena, le cinquième A340 a été repris par Lufthansa, puis par Air Namibia depuis 2006. L'Insu-CNRS et le FZJ paient les coûts de transport de l'instrumentation à la compagnie Air 
Namibia. Actuellement, les trois avions restants transportent le rack Mozaic avec la mesure de $\mathrm{O}_{3}, \mathrm{H}_{2} \mathrm{O}$ et de $\mathrm{CO}$, et l'un de ces trois avions (Lufthansa) transporte l'instrument $\mathrm{NO}_{\mathrm{y}}$.

Les mesures sont échantillonnées toutes les quatre secondes entre le décollage et l'atterrissage, sauf pour l'instrument $\mathrm{NO}_{\mathrm{y}}$, qui ne fonctionne pas pendant le décollage et qui est stoppé dans la dernière phase de l'atterrissage. Après le dépouillement et les contrôles de qualité des observations par les laboratoires en charge des mesures, les données sont stockées à Météo-France. Des données connexes pour l'aide à l'interprétation des observations sont rajoutées par Météo-France en utilisant les analyses du CEPMMT, telles que les rétro-trajectoires de masses d'air le long des routes des avions et la position de la tropopause définie suivant un seuil de tourbillon potentiel. Les utilisateurs ont accès à la banque de données en signant un protocole d'utilisation (http://mozaic. aero.obs-mip.fr/web/). Actuellement, la banque de données est reconstruite en une base de données avec le support du Pôle Ether (Centre for Atmospheric Chemistry Products and Services; http://ether.ipsl.jussieu.fr) : Centre national d'études spatiales (CNES) et Insu-CNRS. Ce nouvel outil offrira les fonctionnalités modernes des bases de données, telles que, par exemple, la sélection des données suivant des critères géographiques, temporels ou de seuil. La base de données intégrera plus tard les observations Iagos.

\section{Sélection \\ de résultats de Mozaic}

\section{Humidité relative de la haute troposphère}

Mozaic a fourni la première climatologie de l'humidité relative de la haute troposphère (HT) et a montré que la HT est plus humide qu'on ne le pensait (Gierens et al., 1997 ; Luo et al., 2007a). C'est un résultat très important car la vapeur d'eau est le principal gaz à effet de serre et parce que ses changements de phase participent à la dynamique atmosphérique. Luo et al. (2007b) montrent que le cycle annuel de la vapeur d'eau dans la HT, dans les analyses du CEPMMT, a un biais général sec de l'ordre de $10 \%$ à $30 \%$ pour l'humidité relative par rapport à la glace (figure 4). La distribution bimodale de la vapeur d'eau dans la HT tropicale observée par Mozaic (Kley et al., 2007)
Figure 3a, 3b, 3c, 3d - Les A340 du programme Mozaic des compagnies aériennes Lufthansa Air France, Austrian (C Takko) et Air Namibia (C Lukas A. Lusser).

a, dans les analyses du CEPMMT, son mode humide, qui est abruptement interrompu en raison de l'absence de processus de sursaturation par rapport à la glace dans le modèle de prévision du CEPMMT. La sursaturation par rapport à la glace est associée à l'existence de cirrus subvisibles, qui ont un impact radiatif important et qui sont fondamentaux pour la formation et la persistance des traînées de condensation des avions subsoniques. Les modèles climatiques ont des difficultés pour représenter correctement la distribution de l'humidité relative. Les fluctuations de température et d'humidité relative mesurées par Mozaic, combinées avec des critères locaux de formation et de persistance des traînées de condensation, ont permis de paramétriser la couverture fractionnaire maximale des traînées de condensation dans la grille d'un modèle (Gierens et al., 1997, 1999, 2000a, 2000b).

\section{Structure en couches \\ de la troposphère,} stratification et mélange

Un résultat principal de Mozaic a été la documentation de la couverture globale et saisonnière de la structure en couches de la troposphère et des fréquences de l'origine de ces strates dans la stratosphère et dans la couche limite planétaire. À peu près la moitié des strates sont relativement riches en ozone et sèches, très probablement d'origine stratosphérique (Newell et al., 1999 ; Thouret et al., 2000). Le cycle saisonnier de la stratification troposphérique en ozone montre clairement un maximum d'été (Colette et al., 2005a, 2005b). La persistance des intrusions stratosphériques est très sensible à l'étirement par cisaillement horizontal, tandis que le mélange des strates provenant de la couche limite planétaire est gouverné

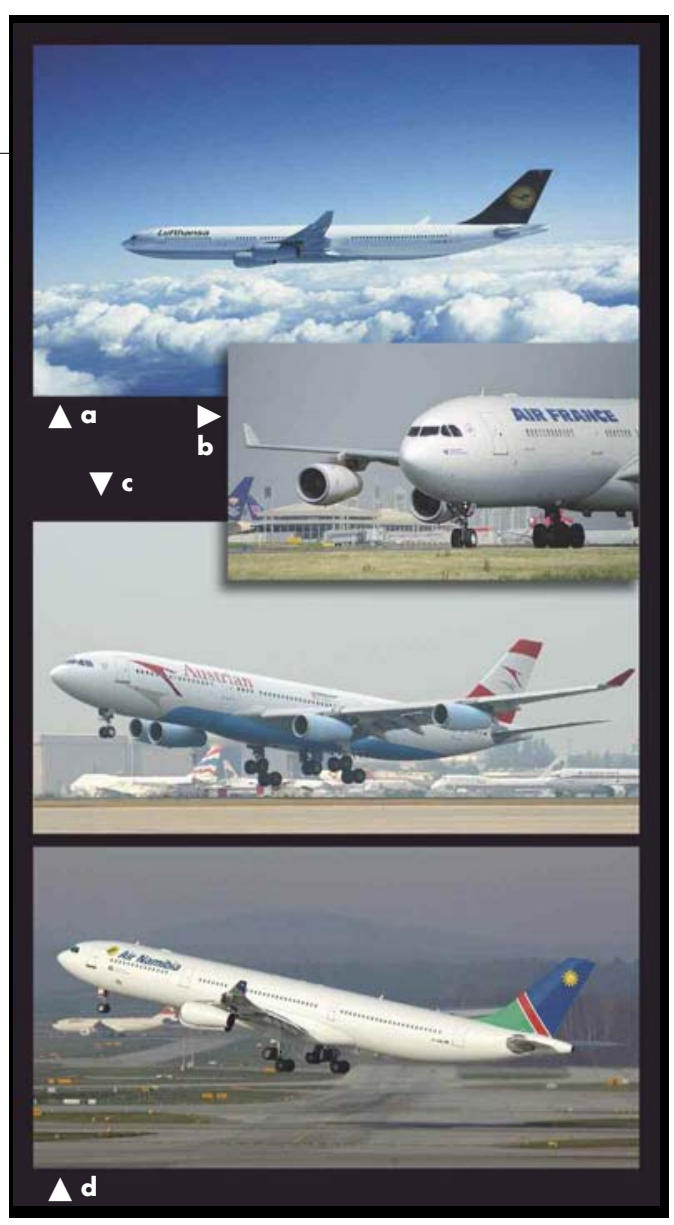

par la convection, surtout en été (Colette et Ancellet, 2006). Les fluctuations de vent et de température mesurées par Mozaic ont été utilisées pour étudier les ondes de gravité et la turbulence bidimensionnelle, et plus généralement le spectre d'énergie cinétique turbulente de l'atmosphère (Cho et al., 2000 ; Cho et Lindborg, 2001 ; Lindborg, 1999 ; Lindborg et Cho, 2000, 2001).

\section{Distributions saisonnières dans la HTBS}

Bien que les distributions des observations Mozaic ne conduisent pas à des climatologies au sens d'un état moyen obtenu sur une grande période de temps ( $>20$ ou 30 ans), la fréquence

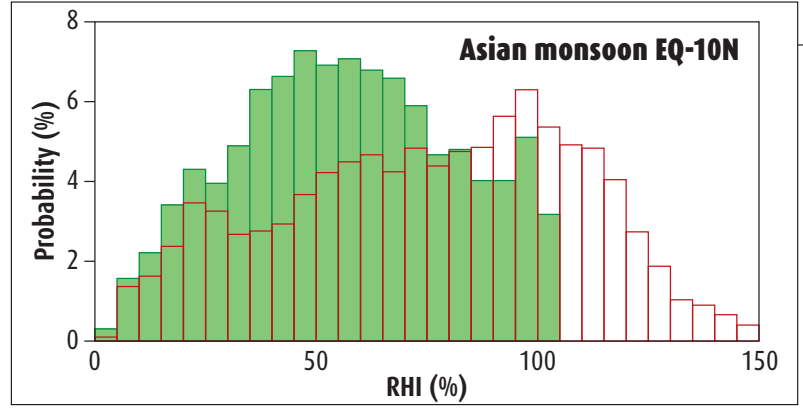

Figure 4 - Fonction de densité de probabilité de l'humidité relative par rapport à la glace dans la haute troposphère (c'est-à-dire $\mathrm{P}<290 \mathrm{hPa}$ ) à partir des observations Mozaic (en rouge) et des analyses du CEPMMT (en vert) sur la région de la mousson asiatique (Luo et al., 2007b). 
Figure $5 a$ et $5 b$ - Distributions moyennes de juin à août de l'ozone

(a), en ppbv, et du monoxyde de carbone (b), en ppbv

établies avec les mesures Mozaic pour la période 2001-2002 Voir Thouret et al. (2006) pour la méthodologie.

des observations, les fines résolutions verticales et horizontales des observations, et les cinq niveaux de vol isobares entre 9 et $12 \mathrm{~km}$ fournissent une image pertinente des distributions globales des espèces et nous permettent d'étudier les cycles saisonniers des espèces depuis le sol jusqu'à la basse stratosphère (BS). Les distributions saisonnières de l'ozone dans la zone HTBS (figure 5) fournissent une description détaillée du changement de phase des cycles saisonniers à travers la tropopause des régions des moyennes latitudes (Thouret et al., 1998a ; Emmons et al., 2000 ; Thouret et al., 2006) et des régions tropicales (Bortz et al., 2006). Les variations saisonnières dans les profils verticaux ont été établies sur 1'Europe, les États-Unis et le Japon (Zbinden et al., 2006), sur la Méditerranée de 1'Est (Kalabokas et al., 2007), sur Pékin en Chine (Ding et al., 2007), sur l'Afrique (Diab et al., 2003 ; Sauvage et al., 2005) et sur le Moyen-Orient (Li et al., 2001).

La climatologie de $\mathrm{NO}_{\mathrm{y}}$ dans la région HTBS montre un cycle saisonnier prononcé et généralement en phase avec celui de l'ozone. Pendant l'hiver, les rapports de mélange de $\mathrm{NO}_{\mathrm{y}}$ dans la région HTBS sont usuellement bien plus faibles que $0,5 \mathrm{ppb}$ et sans

Figure 6 - Distribution estivale moyenne de NOy (ppb) dans la haute troposphère à partir des mesures Mozaic de 2001 à 2005, moyennes sur des grilles de $1^{\circ} \times 1^{\circ}$ pour des observations faites au-dessus de $8 \mathrm{~km}$ d'altitude et $15 \mathrm{hPa}$ au-dessous de la tropopause dynamique c'est-à-dire $\mathrm{P}>\mathrm{P}(2 \mathrm{pvu})+15 \mathrm{hPa}$.

b
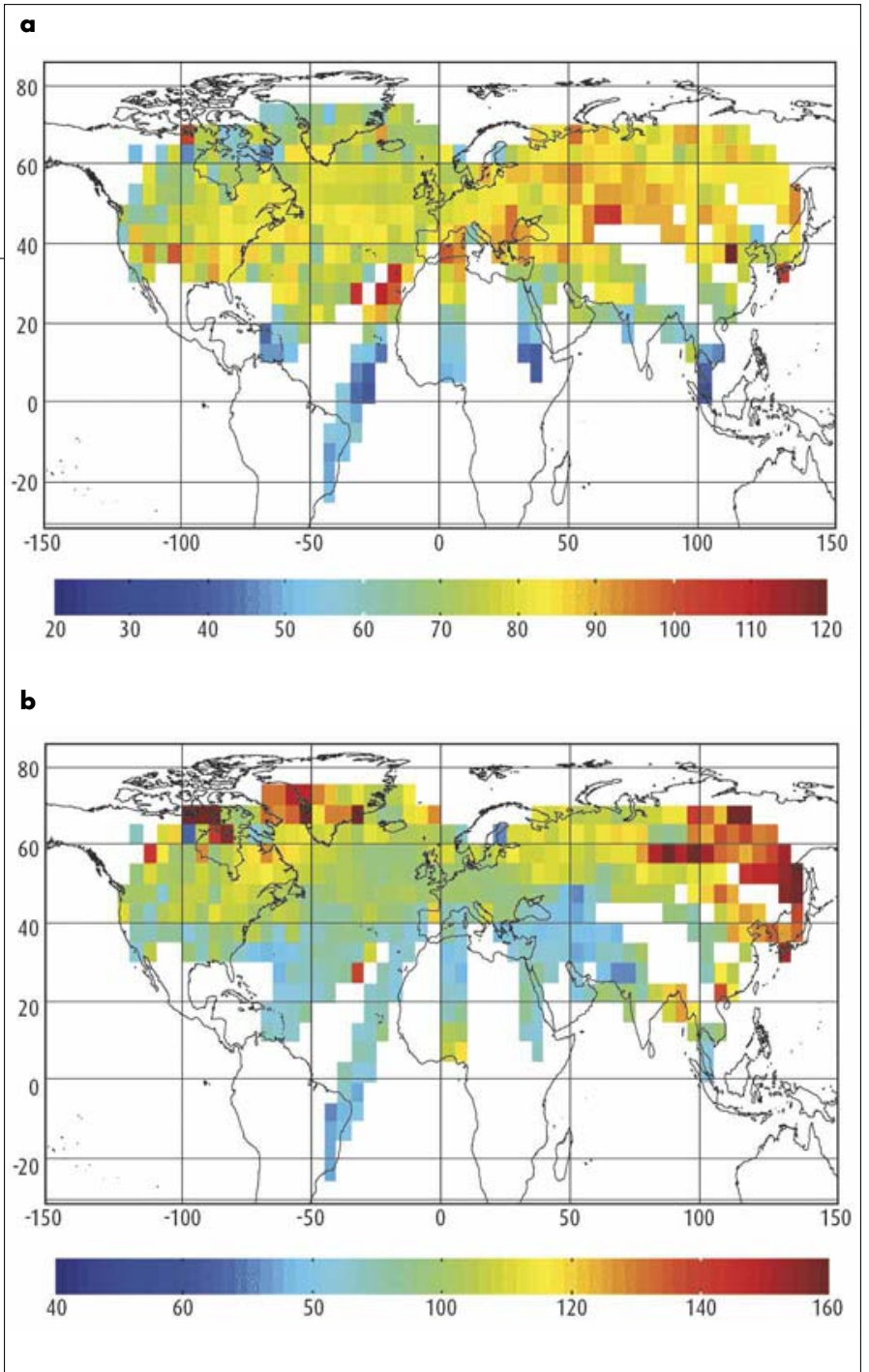

aucune augmentation significative sur les corridors de vol de l'Atlantique nord. De forts rapports de mélange de $\mathrm{NO}_{\mathrm{y}}$ sont observés au printemps et en été, particulièrement sur les côtes est nord-américaines, mais aussi sur l'Europe et l'Asie (figure 6). La corrélation avec $\mathrm{O}_{3}$ et $\mathrm{CO}$ suggère qu'une large fraction de ces valeurs fortes est due à la formation de $\mathrm{NO}_{\mathrm{x}}$ par les éclairs, en plus des contributions éventuelles du transport convectif de sources depuis la couche limite.

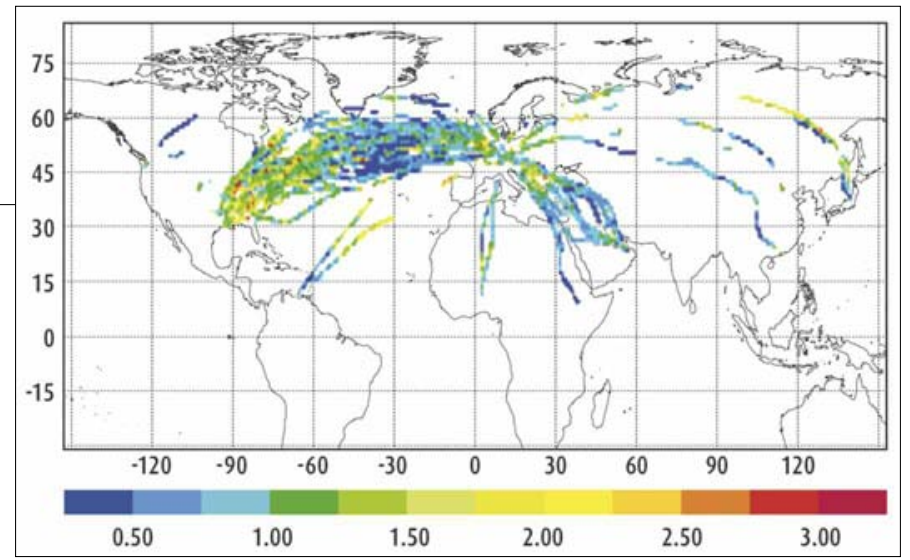

Évaluation

des modèles et assimilation des données

Les modèles globaux de chimie-transport (MCT) d'aujourd'hui sont utilisés pour évaluer les impacts présents et futurs des activités humaines et pour fournir les informations nécessaires aux industriels et décideurs politiques. Ces MCT ont encore des faiblesses dans la représentation des processus qui gouvernent le bilan de l'ozone troposphérique, comme les échanges stratosphère-troposphère, la production photo-chimique nette de la troposphère et la déposition en surface. Les observations Mozaic ont permis d'identifier des erreurs dans la caractérisation des sources d'émissions, des hypothèses erronées sur l'intensité de la convection nuageuse, et des réactions chimiques manquantes dans les modules de chimie des modèles (Law et al., 1998, 2000 ; Emmons et al., 2000 ; Bregman et al., 2001). L'évaluation par les données Mozaic a permis d'évaluer les flux d'échanges stratosphère-troposphère des MCT (Clark et al., 2007) et de mieux évaluer les termes de la production photo-chimique nette (Crowther et al., 2002). On montre que l'assimilation des données d'ozone de Mozaic dans les MCT améliore les résultats des modèles jusqu'à 3-4 jours après la phase d'assimilation (Cathala et al., 2003) et réduit les biais dans les distributions (Chai et al., 2007). Mozaic fournit aussi un des flux de données les plus importants pour évaluer les simulations couplées du modèle de prévision dynamique du CEPMMT avec des MCT dans le projet européen GEMS (Global and Regional Earth-System Atmosphere Monitoring using Satellite and in situ data ; http:// www. ecmwf.int/research/ EU_projects/ GEMS/), qui prépare la construction du Service atmosphérique de GMES (Global Monitoring of the Environment and Security). 


\section{Échanges}

stratosphère - troposphère

Dans les tropiques, Mozaic a montré que les processus d'échanges stratosphère-troposphère, précédemment considérés comme peu importants par rapport aux latitudes moyennes, sont non négligeables et à même d'augmenter la capacité oxydante de la HT tropicale (Cammas et al., 1998 ; Baray et al., 2003 ; Scott et al., 2001). Alternativement, il est montré que le transport troposphère-stratosphère sur la bordure nord de l'anticyclone audessus de la mousson indienne en été peut humidifier la basse stratosphère extratropicale (Dethof et al., 1999). Aux moyennes latitudes, les processus de foliation de tropopause et de formation de gouttes froides d'altitude associés à la dynamique du courant-jet polaire sont des processus relativement bien connus après des campagnes d'observation dédiées. La valeur ajoutée de Mozaic à ces latitudes est l'abondance des observations $\left(\mathrm{O}_{3}, \mathrm{H}_{2} \mathrm{O}\right.$ et $\left.\mathrm{CO}\right)$ dans la région HTBS et le long des profils verticaux qui permet de conduire des cas d'études (Gouget et al., 2000 ; Brioude et al., 2006), de valider des études de bilan pour des modèles mésoéchelles et globaux (Clark et al., 2007) et de conduire d'autres méthodologies (statistique, transport lagrangien) pour évaluer le degré d'irréversibilité des échanges et le mélange qui en résulte (Morgenstern et Carver, 2001 ; Borchi et Marenco, 2002 ; Borchi et al., 2005 ; Brioude et al. 2008).

\section{Variabilité interannuelle et tendances décadaires}

Bien qu'il soit toujours encore trop tôt pour une évaluation significative des tendances, des résultats intéressants émergent actuellement de l'analyse de plus d'une décennie d'observations Mozaic. Une augmentation linéaire de l'ozone d'environ 1 ppbv/an est observée dans les tropiques nord (Bortz et al., 2006). Les fortes concentrations d'ozone observées en 1998-1999 aux moyennes latitudes et dans les régions HT et BS sont très probablement attribuables à une combinaison de différents processus impliquant des modes grande échelle de la variabilité atmosphérique comme 1'Oscillation de NordAtlantique, et du transport de pollutions impliquant les échelles locales à globales (Thouret et al., 2006 ; Zbinden et al., 2006). Les échanges stratosphèretroposphère ont été montrés comme le facteur principal de la variabilité interannuelle marquée de l'ozone pendant quatre saisons hivernales (Morgenstern et al., 2000). Enfin, les profils d'ozone obtenus par Mozaic sur Pékin entre 1995 et 2005 (figure 7) indiquent clairement la détérioration de la qualité de l'air sur cette région de l'Asie (Ding et al., 2007).

\section{Transport des masses d'air enrichies en ozone}

Selon des évaluations de comités internationaux : IPCC (Inter-governmental Panel on Climate Change) et TF HTAP (Task Force on Hemispheric Transport of Air Pollution ; http://www.htap.org/ 10_2007/meeting.htm), l'influence de masses d'air polluées et transportées sur de grandes distances est une des sources d'incertitudes restantes pour l'évaluation et la prévision de la qualité de l'air. La pertinence des observations Mozaic a permis d'améliorer nos connaissances sur les liens entre la distribution des espèces, les sources d'émissions, et les processus atmosphériques de chimietransport. Les études menées avec les données Mozaic documentent :

- l'approvisionnement en ozone dans la HT par les couches limites continentales de l'Amérique du Nord et de l'Eurasie au printemps et en été (Stohl et al., 2001 ; Cooper et al., 2005, 2006) et par la couche limite continentale africaine en automne et en hiver (Stohl et al., 2001) ;

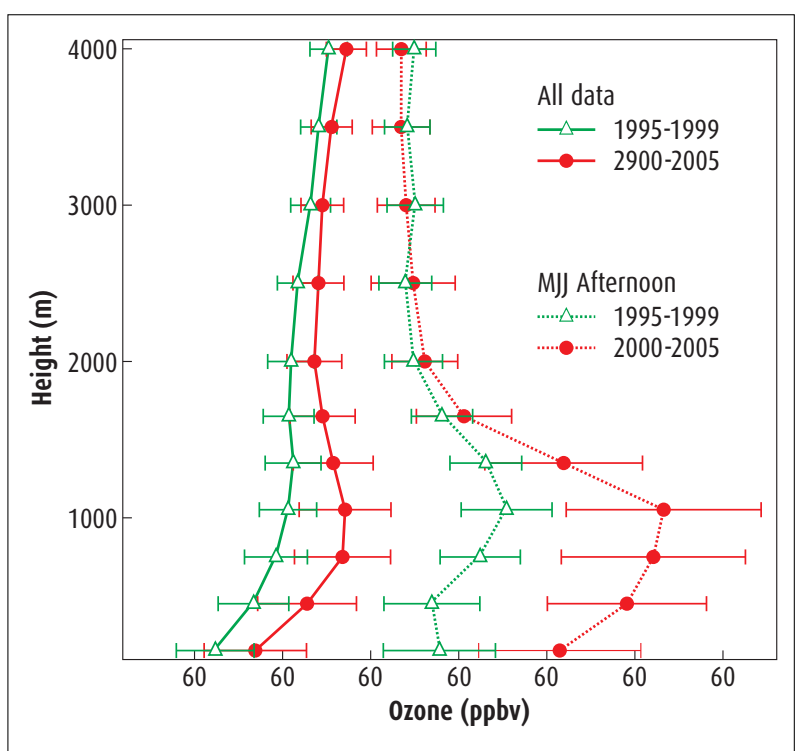

- le transport de panaches de feux de forêt par la convection et par les ceintures de transport d'air chaud des dépressions [figure 8] (Nédélec et al., 2005) ;

- la canalisation de la pollution par des régimes atmosphériques persistants sur la Méditerranée (Kalabokas et al., 2007);

- le transport de la pollution et des panaches de feux de forêt par l'harmattan et les alizés en Afrique (Sauvage et al., 2005, 2007c) et par les cellules de transport de Hadley et de Walker, en incluant les panaches enrichis en $\mathrm{NO}_{\mathrm{x}}$ par l'activité électrique dans la convection tropicale (Sauvage et al., 2006, 2007a, 2007b).

Figure 7 - Comparaison des profils moyens d'ozone dans la basse troposphère $(0-4 \mathrm{~km})$ sur Pékin de 1995 à 1999 (en vert) et de 2000 à 2005 (en rouge). Les lignes continues sont des moyennes annuelles et les lignes pointillées sont des moyennes sur la période d'été (mai à juillet) entre $15 \mathrm{~h}$ et $16 \mathrm{~h}$ locales.

Figure 8 - Concentrations de CO (en ppbv) au-dessus de $8 \mathrm{~km}$ d'altitude sur l'Asie entre $40^{\circ} \mathrm{N}$ et $65^{\circ} \mathrm{N}$, pour huit jours de juin 2003. La zone ombrée en bleu indique le niveau moyen saisonnier du C0 à 110 ppbv. (Nédélec et al., 2005)

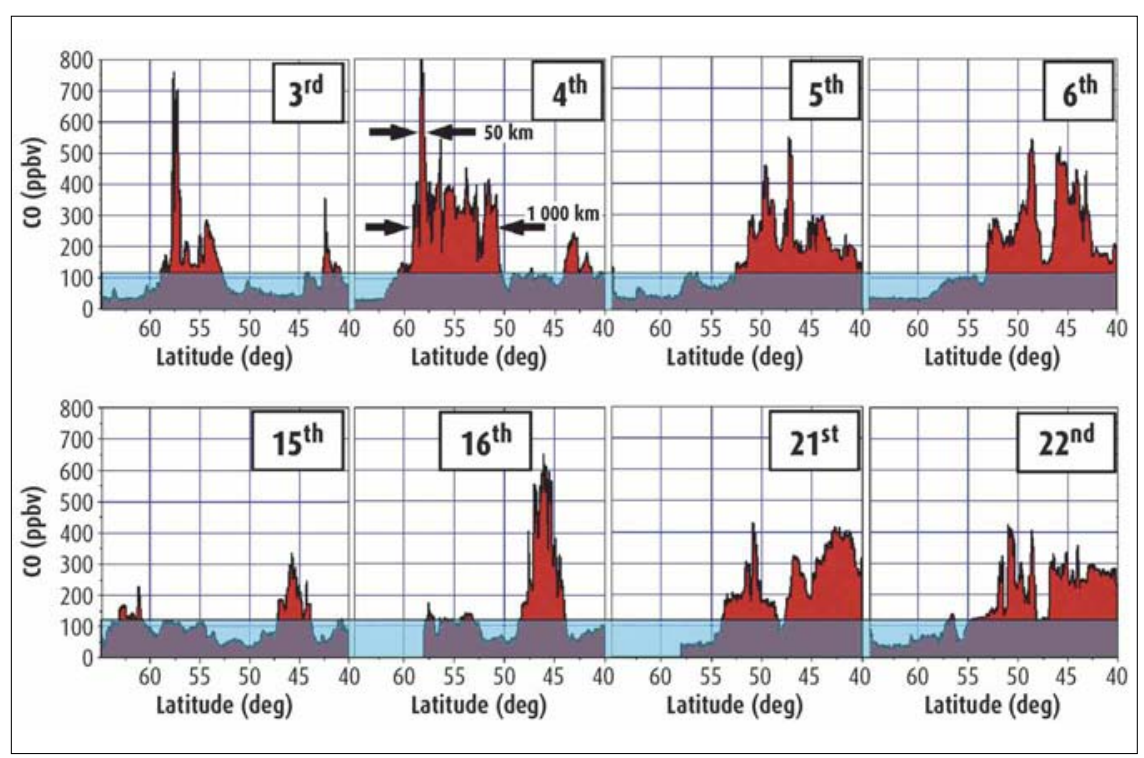




\section{Couche limite planétaire} et qualité de l'air régionale

Bien que le programme Mozaic n'ait pas été initialement monté pour étudier les processus gouvernant la distribution de l'ozone dans la couche limite planétaire et la qualité de l'air au niveau régional, la combinaison des données Mozaic avec des données de station de surface est appropriée pour ce type d'études. Avec un réseau de stations de montagnes couvrant la partie ouest de l'Europe entre 115 et 3580 mètres d'altitude, Chevalier et al. (2007) montrent que les mesures de surface capturent statistiquement la stratification de l'ozone révélée par Mozaic à Francfort (Allemagne), pointant ainsi sur le rôle de l'altitude et du transport vertical rapide dans l'interprétation des mesures d'ozone sur des stations de montagne. Lopportunité, pendant la vague de chaleur de l'été 2003, d'avoir trois avions Mozaic basés à Francfort, a permis de définir précisément les anomalies d'ozone et de monoxyde de carbone dans la couche limite (Tressol et al., 2007), ce qui permet de cerner les difficultés auxquelles font face les modèles globaux de chimie-transport et les modèles régionaux pour reproduire ces anomalies.

\section{Validation de produits satellitaires}

Bien que les capteurs satellitaires fournissent une couverture quasi globale des observations, les données souffrent encore d'un manque de précision et de résolution verticale et nécessitent d'être validées à intervalles réguliers avec des mesures in situ plus précises. Les données Mozaic sont utilisées pour la validation des inversions de radiances en profils verticaux de rapports de mélange de gaz trace pour un nombre croissant de capteurs, par exemple, la vapeur d'eau avec POAM III [Polar Ozone and Aerosol Measurement] (voir Nedoluha et al., 2002 ; Prados et al., 2003), le CO avec l'instrument Mopitt (Measurements of Pollution in the Troposphere) sur le satellite Terra (Emmons et al., 2007), l'ozone et le CO avec l'instrument MLS (Microwave Limb Sounder) à bord de Aura (Livesey et al., 2007), le CO de l'instrument ACE à bord de FTS (Clerbaux et al., 2007) et la vapeur d'eau et la température par radiooccultation GPS (Heise et al., 2007). Dans les tropiques, la cohérence de la détection des flashs des éclairs et des distributions de précurseurs de l'ozone (colonnes $\mathrm{NO}_{2}$ ) avec les instruments Gome, OTD et LIS ont été évaluées en utilisant un MCT et les mesures Mozaic (Sauvage et al., 2007b).

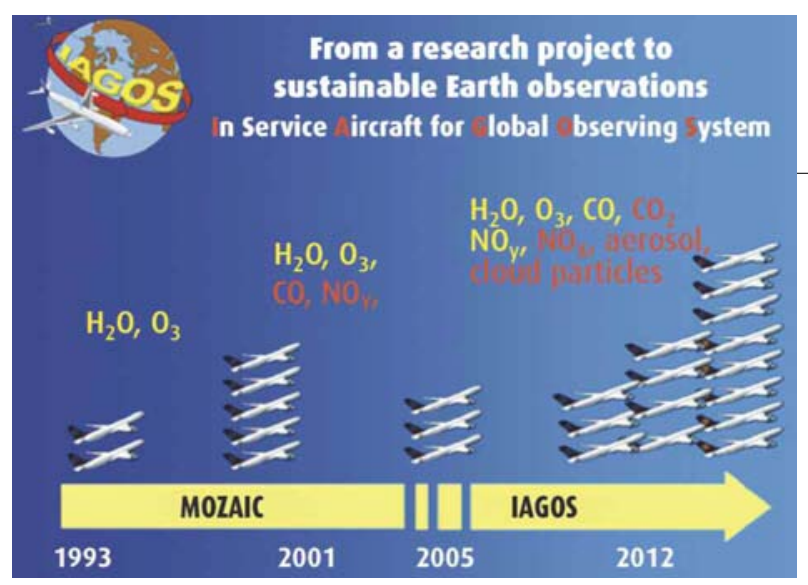

Figure 9 - Schéma temporel du déploiement des avions instrumentés des programmes Mozaic et lagos.

Impact des émissions

des avions subsoniques sur l'ozone

Les mesures d'ozone par des programmes comme Mozaic constituent une source de référence avec laquelle tester les simulations d'impact sur la composition atmosphérique des émissions de $\mathrm{NO}_{\mathrm{x}}$ par les avions subsoniques. Ces études ont été faites dans plusieurs programmes européens à l'aide des données Mozaic, comme Tradeoff (Isaksen et al., 2003) et Scenic (Rogers et al., 2005), et continuent dans le programme actuel Quantify (http://www.pa.op.dlr. de/quantify/). Au cours des années 1990 , la fourchette des perturbations prévues sur l'ozone s'est affinée dans les différents groupes internationaux de modélisation des impacts du transport aérien (Brasseur et al., 1998). Le groupe de référence de l'université d'Oslo (Gauss et al., 2006) prévoit en moyenne zonale des augmentations maximales d'ozone produites par les émissions d'avion de 3,1 ppbv en septembre à 7,7 ppbv en juin dans la région de la tropopause de l'hémisphère nord. La validation de leurs simulations leur permet de discuter des avantages et des inconvénients des stratégies d'atténuation envisageables, telles que le réglage de l'altitude de vol ou des impacts des émissions sur de nouvelles routes aériennes comme les routes polaires.

\section{lagos-ERI}

Après le succès du programme de recherche Mozaic, il s'est agit de recapitaliser les points forts de ce programme et d'évoluer vers une infrastructure de recherche pérenne. L'initiative européenne Iagos-ERI cherche à construire une infrastructure distribuée et durable, pour des observations globales de la composition atmosphérique, à partir d'une flotte nationales (figure 9).
Iagos-ERI est une des nouvelles infra-

d'avions commerciaux. L'objectif est d'installer progressivement des instruments automatiques à bord de dix à vingt avions long-courrier de plusieurs compagnies aériennes interstructures de recherche inscrites sur la feuille de route 2006 du forum européen ESFRI (European Strategy Forum for new Research Infrastructures ; http://cordis.europa. eu/esfri/). Cette initiative est née du programme européen Mozaic. Des liens de collaboration ont déjà été établis avec d'autres programmes de mesures aéroportées de routine. Caribic (Brenninkmeijer et al., 2007), l'autre programme européen, très complémentaire de Mozaic et qui embarque deux fois par mois un container de 1,5 tonne dans un avion cargo long-courrier de Lufthansa, fait ainsi maintenant partie de l'infrastructure de recherche Iagos-ERI. Il est aussi prévu, dans les prochaines années, d'établir des liens privilégiés avec le programme japonais Contrail (Machida et al., 2007). L'objectif final est de construire la composante avion du système d'observations global Igaco (Integrated Global Atmospheric Chemistry Observations System ; Barrie et al., 2004).

Iagos-ERI fournira des observations in situ de haute qualité de gaz à effet de serre, de gaz réactifs, d'aérosols et d'hydrométéores nuageux dans la région HTBS qui est l'une des régions les plus sensibles vis-à-vis du changement climatique et qui nécessite d'être observée de façon adéquate. En même temps, Iagos-ERI fournira des profils détaillés de la troposphère, qui sont de la plus haute importance pour prévoir les changements dans la qualité de l'air aux échelles locales et régionales, et pour comprendre leurs causes. L'utilisation des avions commerciaux dans la région HTBS, où d'autres techniques de mesure (par exemple, les satellites) ont encore des limitations techniques, autorise en effet la collecte d'observations hautement pertinentes, sur des échelles spatiotemporelles et avec des fréquences impossibles à atteindre avec des avions de recherche. 


\section{La justification}

Le changement climatique global est sans conteste un des problèmes environnementaux majeurs auquel fait face l'humanité aujourd'hui, avec des implications sur la stabilité politique et l'économie globale. Des prévisions fiables du climat futur à l'aide de modèles climatiques constituent une exigence centrale et fondamentale pour déterminer les futures stratégies d'atténuation. Cependant, les rapports d'évaluations internationaux (IPCC, WMO-Unep, Igac, Sparc et Eurotrac-2) montrent que les plus grandes incertitudes dans l'estimation du changement climatique et de la qualité de l'air persistent dans :

- l'influence de la vapeur d'eau, des aérosols et des nuages sur le climat, en particulier dans la HT ;

- l'effet indirect des aérosols sur le climat via les influences sur la formation des nuages et leur temps de vie ;

- l'influence de l'ozone et des aérosols transportés sur de grandes distances sur la qualité de l'air et le climat ;

- les changements climatiques induits par les changements dans les échanges stratosphère-troposphère, avec des implications sur les bilans de l'ozone et de la vapeur d'eau et avec d'importantes rétroactions sur la qualité de l'air et du climat ;

Tableau 1 - Domaines clés définis par Igaco et les espèces à mesurer (Barrie et al., 2004). La dernière colonne montre les espèces mesurées par lagos (en fond rouge les espèces mesurées de façon moins fréquente par la composante Caribic de lagos).

\begin{tabular}{|c|c|c|c|c|c|}
\hline Espèces chimiques & $\begin{array}{l}\text { Qualité } \\
\text { de l'air }\end{array}$ & $\begin{array}{l}\text { Capacité } \\
\text { oxydante }\end{array}$ & Climat & $\begin{array}{l}\text { Diminution } \\
\text { de l'ozone } \\
\text { strato- } \\
\text { sphérique }\end{array}$ & lagos \\
\hline $\mathrm{O}_{3}$ & $\nabla$ & $\nabla$ & $\nabla$ & $\nabla$ & $\nabla$ \\
\hline $\mathrm{CO}$ & $\nabla$ & $\nabla$ & - & - & $\nabla$ \\
\hline $\mathrm{J}\left(\mathrm{NO}_{2}\right)$ & $\nabla$ & $\nabla$ & - & - & \\
\hline $\mathrm{J}\left(\mathrm{O}_{1} \mathrm{D}\right)$ & $\nabla$ & $\nabla$ & - & - & \\
\hline $\mathrm{H}_{2} \mathrm{O}$ (vapeur d'eau) & $\nabla$ & $\nabla$ & $\nabla$ & $\nabla$ & $\nabla$ \\
\hline $\mathrm{HCHO}$ & $\nabla$ & $\nabla$ & - & - & \\
\hline COV & $\nabla$ & $\nabla$ & - & - & $\nabla$ \\
\hline Oxydes d'azote : $\mathrm{NO}_{\mathrm{x}}$ & $\nabla$ & $\nabla$ & - & $\nabla$ & $\nabla$ \\
\hline Espèces réservoir : $\mathrm{HNO}_{3}$ & $\nabla$ & $\nabla$ & - & $\nabla$ & $\nabla$ \\
\hline $\mathrm{N}_{2} \mathrm{O}$ & - & - & $\nabla$ & $\nabla$ & $\nabla$ \\
\hline $\mathrm{SO}_{2}$ & $\nabla$ & - & $\nabla$ & - & \\
\hline $\begin{array}{l}\text { Halogènes actifs : } \mathrm{BrO}, \mathrm{ClO} \\
\text { Réservoirs : } \mathrm{OClO}, \mathrm{HCl}, \mathrm{ClONO} 2 \\
\text { Sources : } \mathrm{CH}_{3} \mathrm{Br}, \mathrm{CFC}-12, \\
\text { HCFC-22, halons }\end{array}$ & $\begin{array}{l}\square \\
\nabla \\
\nabla\end{array}$ & $\begin{array}{l}- \\
- \\
-\end{array}$ & $\begin{array}{l}- \\
- \\
-\end{array}$ & $\begin{array}{l}\square \\
\square \\
\square\end{array}$ & $\begin{array}{l}\square \\
\square\end{array}$ \\
\hline Propriétés des aérosols & $\nabla$ & - & $\nabla$ & $\nabla$ & $\nabla$ \\
\hline $\mathrm{CO}_{2}$ & - & - & $\nabla$ & - & $\nabla$ \\
\hline $\mathrm{CH}_{4}$ & - & $\nabla$ & $\nabla$ & $\nabla$ & $\nabla$ \\
\hline
\end{tabular}

précisément aux priorités définies dans le rapport Igaco comme le montre le tableau 1. Le rapport Igaco recommande spécifiquement les mesures de dioxyde de carbone à partir de réseaux de mesure in situ incluant des observations aéroportées. Des profils routiniers de $\mathrm{CO}_{2}$ par mesures in situ sont nécessaires pour la validation des colonnes et des profils de cette espèce par des moyens satellitaires, par exemple avec OCO, Airs, Sciamachy et Iasi. L'importance des mesures de routine par avion a été explicitement reconnue par le rapport Gato de GMES, la contribution européenne de GEO (Braathen et al., 2004).

qui à leur tour empêchent des visions précises du changement climatique et de ses causes. Le besoin de continuer et d'étendre les programmes d'observations actuels est spécifiquement exprimé. Le rapport Igaco [Integrated Global Atmospheric Chemistry Observations] (voir Barrie et al., 2004), qui est maintenant adopté par l'initiative GEO (Global Earth Observation), recommande spécifiquement la continuation et l'extension des mesures de routine par avion comme un complément essentiel aux observations par des moyens sol et des satellites. Iagos-ERI servira à mettre en service la composante avion de Igaco. Les mesures proposées dans Iagos correspondent

\section{Le concept technique}

Le concept technique de Iagos est fondé sur l'expérience acquise dans le programme Mozaic. En 1994, des systèmes de mesure de l'ozone et de la vapeur d'eau, développés par le Laboratoire d'aérologie et le FZJ, ont été installés dans les soutes avioniques de cinq Airbus A340. L'installation a été faite sur des avions neufs dans les chaînes de montage de l'usine toulousaine. L'instrumentation Mozaic a été modifiée en 2001 pour ajouter les mesures de $\mathrm{CO}$ sur les cinq avions et les mesures de $\mathrm{NO}_{\text {y }}$ sur un avion. Mozaic ayant été initialement conçu comme un programme de recherches sur trois ans, plusieurs difficultés techniques sont devenues apparentes sur l'opération des systèmes après la phase initiale. La maintenance de l'instrumentation n'est possible qu'en étroite collaboration avec le service de maintenance des compagnies aériennes. Un autre problème significatif est que l'étagère de l'instrumentation Mozaic (figure 2) est assez lourde $(134 \mathrm{~kg}+$ $50 \mathrm{~kg}$ pour l'instrument $\mathrm{NO}_{\mathrm{y}}$ ) et encombrante, et que l'emplacement original d'implémentation de l'instrumentation Mozaic n'est plus disponible dans les A340 récents. Aucune disposition n'avait été prise pour inclure dans les Bulletins de service d'Airbus les procédures pour installer l'instrumentation Mozaic sur un A340 déjà en service. Après treize années de service, les avions équipés Mozaic risquent donc d'être revendus sans qu'il soit possible d'établir des collaborations avec la nouvelle compagnie aérienne propriétaire. Les mesures des trois avions restants (deux de Lufthansa et un d'Air Namibia) doivent donc assurer un pont entre la fin du programme Mozaic et le début du programme Iagos-ERI (figure 9). 
À la fin du programme Mozaic en 2003, il était donc clair que, pour une opération durable du programme d'observation, l'instrumentation devait être complètement revue, de façon à réduire le poids ainsi que la taille de plus de $50 \%$ et à obtenir les certificats aéronautiques requis pour l'installation et la maintenance à bord d'avions déjà en service. Cette situation a été le point de départ de Iagos-DS (Integration of Measurements of Aircraft into a Global Observing System - Design Study), un projet visant à une étude de conception pour les nouvelles infrastructures de recherches européennes, cofinancé par le sixième Programme cadre de recherche et développement technologique (PCRDT) européen de 2005 à 2009, et sous la coordination du centre de recherche de Jülich (FZJ). Un tâche principale de Iagos-DS sous la responsabilité du Laboratoire d'aérologie est la nouvelle conception de l'instrumentation Iagos de base (appelé « IagosPackage I ») en un seul instrument de $70 \mathrm{~kg}$ (figure 10).

En raison des exigences scientifiques décrites plus haut, le partenariat de Mozaic a été étendu dans Iagos-DS, de façon à augmenter les capacités de mesure avec de nouveaux instruments pour les hydrométéores nuageux (université de Manchester, Royaume-Uni), les aérosols (Institut de physique de l'atmosphère du DLR, Allemagne) et le dioxyde de carbone (MPI-BGC, Jena, Allemagne).

Le concept technique de la nouvelle instrumentation Iagos et son installation dans la soute avionique de l'Airbus A340 sont décrits figure 11. Il comprend deux ensembles instrumentaux :

- package I : $\mathrm{O}_{3}, \mathrm{H}_{2} \mathrm{O}$, CO, hydrométéores nuageux, système d'acquisition et de transmission en temps différé (environ 24-48 heures) pour l'ensemble des paramètres et en temps réel (environ 15 minutes) pour une sélection de paramètres ;

- package IIa : $\mathrm{NO}_{\mathrm{y}}$ (la somme de $\mathrm{NO}_{\mathrm{x}}$ et de ses produits d'oxydation atmosphérique) ;

- package IIb : $\mathrm{NO}_{\mathrm{x}}$;

- package IIc : aérosols ;

- package IId : $\mathrm{CO}_{2}$.

Le package I sera installé sur chaque avion, avec en option un des éléments du package II. Les instruments auront chacun leur certificat aéronautique pour l'installation et l'opération à bord d'un A340 en service, incluant le formulaire « Easa Form One » pour la réinstallation après maintenance. La modifica-

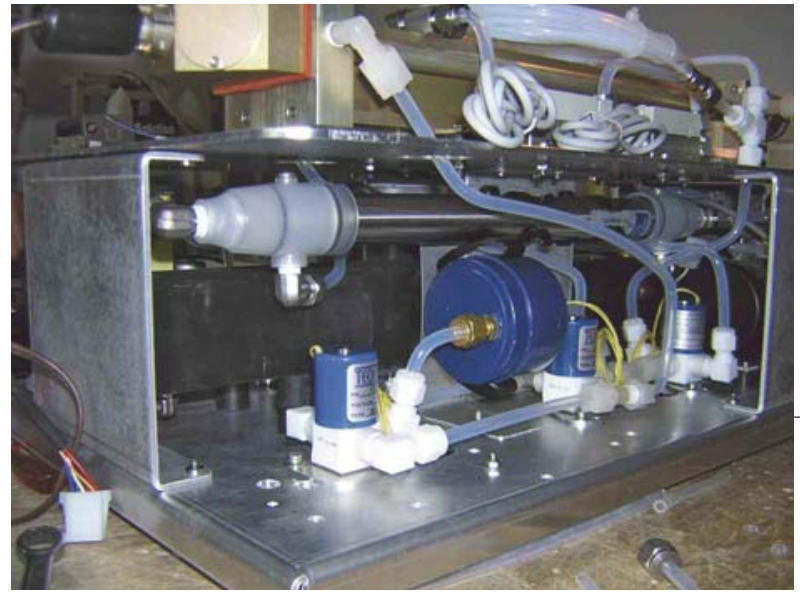

Figure 10 - Le prototype lagos-Package I qui combinera les mesures d'ozone, de monoxyde de carbone, de vapeur d'eau, le comptage des hydrométéores et les systèmes d'acquisition et de transmission des données (responsabilité Laboratoire d'aérologie, en collaboration avec le FZJ et l'université de Manchester).

- transmission en temps réel (résolution d'environ 15 minutes) pour la température, le vent, l' $\mathrm{O}_{3}$, la

tion de structure de l'avion (Supplementary type certificate) est entreprise par le Laboratoire d'aérologie en collaboration avec un partenaire industriel, autorisé à concevoir et exécuter des modifications sur les avions Airbus. Iagos-DS fournira le Bulletin de service d'Airbus pour l'installation et la maintenance des instruments Iagos sur les A340. La modification de structure de l'avion pour l'installation sera effectuée pendant les maintenances prévues des avions, sans coûts d'immobilisation pour les compagnies aériennes.

Iagos développera les procédures de maintenance certifiées pour le remplacement périodique des instruments et pour l'assurance de qualité des mesures. Le programme de maintenance des instruments sera le plus léger possible, environ tous les six mois pour le package I et tous les deux-trois mois pour le package II (en raison du besoin de renouveler les cylindres à gaz et de plus fréquents tests et étalonnages en laboratoire). La transmission automatique des données autorisera une surveillance quotidienne de l'état de marche de l'ensemble de l'instrumentation.

La transmission automatique des données sera faite en deux étapes :

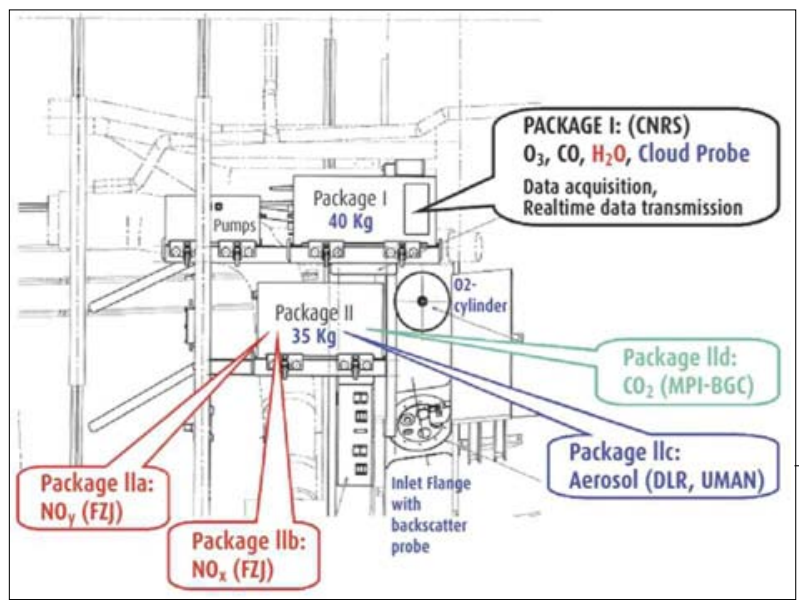

vapeur d'eau et d'autres paramètres à sélectionner suivant les besoins des modèles de prévision météorologiques et de chimie-transport. Ici, une collaboration étroite est en cours entre MétéoFrance et la communauté Amdar (Aircraft meteorological data relay), dont le système est déjà installé sur de nombreux avions ;

- transmission différée (24-48 heures environ depuis l'aéroport vers le Laboratoire d'aérologie) de toutes les données acquises en vol, en utilisant les réseaux de communication déjà établis sur les zones aéroportuaires, tels que le GPRS ou le WiFi.

Avec la transmission en temps réel, les modèles opérationnels pourront recevoir les mesures pour leur validation immédiate ou de leur assimilation. La communauté scientifique disposera des données de Iagos avec très peu de retard sur l'opérationnel via la transmission en temps différé. La nouvelle conception de la base de données de Mozaic, étendue à Iagos, augmentera la disponibilité et l'utilisation des données.

La seconde composante de Iagos-ERI est constituée par le programme Caribic (Brenninkmeijer et al., 2007) qui a rejoint le partenariat de la nouvelle infrastructure. L'A340 cargo équipé du container Caribic (un A340-600 opéré par Lufthansa) est équipé d'un système spécial de prise d'air. Il fournira des mesures sur une longue liste d'espèces chimiques,

Figure 11 - Concept technique pour l'installation des instruments lagos dans I'Airbus A340. 
incluant celles mesurées sur les avions équipés Iagos, mais aussi des mesures de méthane, de $\mathrm{N}_{2} \mathrm{O}$, la famille des hydrocarbures et fluorocarbones, des mesures très détaillées des aérosols, et un système MAX-DOAS pour la mesure par télédétection d'espèces chimiques. Des mesures d'isotopes des constituants en trace incluent celles du ${ }^{14} \mathrm{CO}$, un excellent traceur pour estimer les radicaux $\mathrm{OH}$ au niveau global (Volz et al., 1981 ; Brenninkmeijer et al., 1992). En raison de la plus grande complexité technique des instruments et du coût de transport élevé de l'instrumentation, le container Caribic ne sera déployé que quelques fois par mois.

\section{Où en sommes nous ?}

Dans le projet actuel Iagos-DS, les certificats aéronautiques des instruments révisés de Mozaic sont en cours d'obtention et les nouveaux développements techniques des instruments pour les hydrométéores et les aérosols sont en voie d'achèvement. Pour la mesure automatique du $\mathrm{CO}_{2}$, plusieurs solutions sont en cours d'investigation. Les certifications des nouveaux instruments $\left(\mathrm{CO}_{2}\right.$, aérosols, module de transmission en temps réel) seront obtenues dans la prochaine phase de Iagos qui démarrera en 2008 avec un cofinancement du septième PCRDT, pour une durée de quatre ans et qui servira à établir les conditions légales et logistiques pour l'opération de la nouvelle infrastructure européenne. La composition du projet Iagos-ERI, sous la coordination du FZJ, est décrite dans le tableau 2. Ce nouveau projet permettra par ailleurs de financer les modifications pour automatiser les opérations du container Caribic et pour continuer des travaux de recherche sur le développement d'une future instrumentation encore plus miniaturisée pour Iagos.

Plusieurs compagnies aériennes opérant des long-courriers A340 ont déjà exprimé leur intérêt de participer au projet Iagos-ERI, par exemple,
Lufthansa, Air France, Iberia, South African Airways et China Airlines Taiwan. À terme, une couverture globale incluant l'hémisphère sud et le Pacifique sera établie.

Des efforts majeurs sont encore nécessaires pour sécuriser les supports financiers des phases de construction et d'opération de l'infrastructure. La figure 12 montre l'estimation des coûts totaux pour le déploiement des instruments sur une vingtaine d'A340. L'investissement initial pour les nouveaux instruments, leur certification et la modification des avions est d'environ cinq millions d'euros. L'opération des systèmes, incluant leur maintenance, l'assurance de qualité des données, le développement et la maintenance de la base de données et les coûts de transport éventuels, est estimée à environ 250000 euros par avion et par an.

Une partie significative de ces coûts devra être supportée par les États membres sous forme de contributions

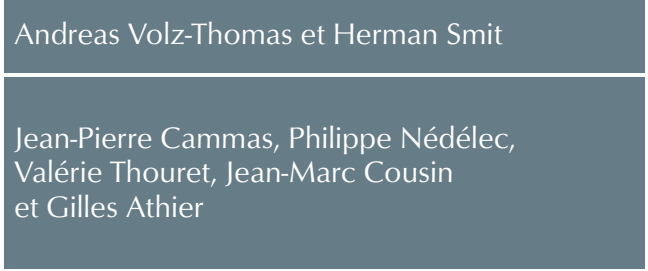

Fernand Karcher et Magali Stoll

Rod L. Jones
Martin Gallagher

Andreas Petzold et Hans Schlager

Christine M. Bickerstaff

Andy Kershaw

Christoph Gerbig

Andreas Waibel

Harald Franke

Markus Herman

Len Barrie

Tracy Henshaw

Françoise Girod

Delphine Texier
Forschungszentrum Jülich

Laboratoire d'aérologie,

Centre national de la recherche scientifique,

Observatoire Midi-Pyrénées

Météo-France,

Centre national de recherches météorologiques

Université de Cambridge

Université de Manchester

Deutsches Zentrum für Luft- und Raumfahrt, Institut für Physik der Atmosphäre

Airbus UK Ltd

British Airways plc

Max-Planck-Institut für Biogeochemie

Deutsche Lufthansa AG

Enviscope $\mathrm{GmbH}$

Leibniz-Institut für Troposphärenforschung

World Meteorological Organization

Natural Environment Research Council

Centre national d'études spatiales

UMS 831, observatoire Midi-Pyrénées

\begin{tabular}{|c|c|}
\hline FZJ & Jülich \\
\hline CNRS & Toulouse \\
\hline MF & Toulouse \\
\hline UCAM & Cambridge \\
\hline UMAN & Manchester \\
\hline DLR & Oberpfaffenhofen \\
\hline AUK & Bristol \\
\hline BA & Hammondsworth \\
\hline MPG & Jena \\
\hline DLH & Cologne \\
\hline $\begin{array}{l}\text { Envi- } \\
\text { scope }\end{array}$ & Francfort \\
\hline IFT & Leibzig \\
\hline WMO & Genève \\
\hline NERC & Londres \\
\hline CNES & Toulouse \\
\hline OMP & Toulouse \\
\hline
\end{tabular}




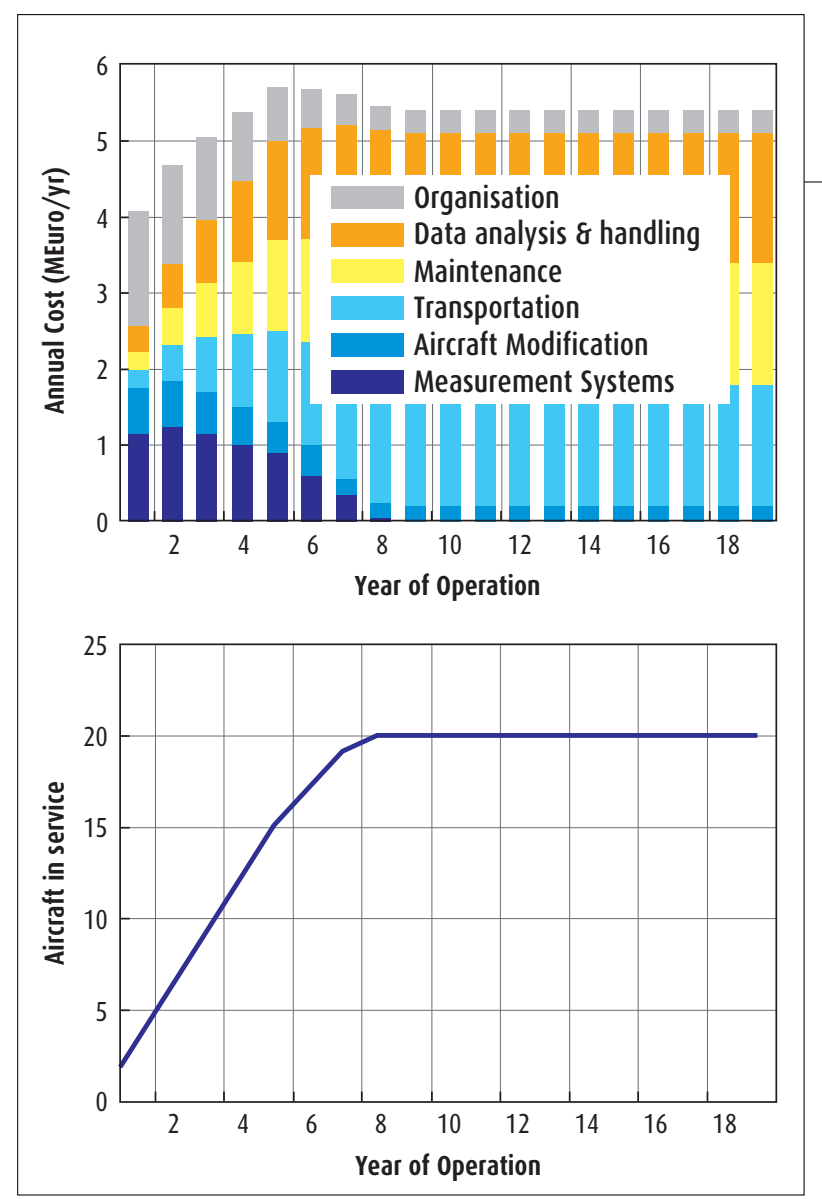

institutionnelles (personnels techniques et scientifiques des instituts partenaires, allocations des espaces de travail en laboratoires, équipements de tests et d'étalonnages...) et gouvernementales (Iagos-ERI est classé comme un très grand instrument au ministère de la Recherche en France). Une autre partie significative des coûts, dont les coûts opérationnels, devront être couverts par d'autres sources, à travers des programmes nationaux et internationaux, comme par exemple le Service atmosphérique de GMES en Europe. La disposition d'un financement récurrent sera le facteur limitant de la taille de la flotte aérienne instrumentée. La sécurisation de ces ressources financières est l'un des objectifs majeurs du nouveau projet Iagos-ERI.

\section{Conclusions}

Au cours de la période 1994-2007, les capteurs Mozaic, embarqués à bord de cinq Airbus A340, ont fourni des données de la plus haute importance pour améliorer nos connaissances sur cette région de la HTBS, qui est par ailleurs encore trop mal observée par les réseaux de sondage et par les moyens satellitaires. Mozaic a démontré la faisabilité de mesures de routine de haute

Figure 12 - Projection de l'estimation des coûts annuels pour l'établissement et l'opération de vingt ensembles instrumentaux par catégorie de coût (en haut) et pour le nombre d'avions en service (en bas).

Le schéma n'inclut pas les coûts pour Caribic qui sont estimés à environ 1 million d'euros par an.

qualité collectées à bord d'avions commerciaux en produisant treize années de mesures qualifiées de l'ozone et de la vapeur d'eau, et sept années de mesures qualifiées du monoxyde de carbone et des oxydes d'azote $\mathrm{NO}_{\mathrm{y}}$. Malgré un budget conséquent initial principalement dédié à la certification aéronautique, le prix de revient moyen des données pour un vol Mozaic (500 euros environ par vol) se compare très favorablement aux coûts des sondages d'ozone par ballon. Avec plus de cent vingt articles (journaux à comité de lecture) utilisant des données Mozaic, le programme a développé de fortes retombées dans la communauté scientifique, bien au-delà de ce qui avait été anticipé à son lancement. Les retombées scientifiques s'inscrivent dans la connaissance des distributions saisonnières des espèces dans la région HTBS, des échanges stratosphère-troposphère, de la structure en couche de la troposphère et du transport à longue distance de la pollution. Les résultats montrent aussi des variabilités interannuelles qui posent question sur leurs origines et les premiers signes de tendances sur la décennie. La base de données Mozaic fournit une profusion d'observations de haute qualité pour la validation des modèles globaux et régionaux de chimietransport, des nouvelles générations d'instruments satellitaires et pour la validation des simulations d'impacts des émissions par les avions subsoniques. La diffusion des résultats a contribué à des réductions significatives des incertitudes sur les estimations du changement climatique, comme il a été reconnu dans plusieurs rapports internationaux (IPCC, WMO-Unep, Igac, Sparc et Eurotrac).

Iagos s'efforce d'intégrer les communautés utilisatrices des données avions et leurs besoins, de façon à créer un système de surveillance durable de l'atmosphère chimique globale. Trente ans après la fin du programme Gasp (Global Atmospheric Sampling Program), le premier programme à avoir utilisé des avions commerciaux comme vecteur de mesure (Nastrom, 1979), Iagos offre la vision de la surveillance routinière de l'atmosphère, où les systèmes d'observations, construits et certifiés selon les normes aéronautiques, peuvent être installés sur des avions long-courrier. Un tel système devrait garantir le plus grand degré de flexibilité et pourrait même aider des pays en voie de développement à s'équiper de moyens de mesure aéroportés à moindre coût. L'assurance de qualité des observations est escomptée à travers des programmes QA-QC mis en place par exemple par l'OMM et le réseau GAW. Si cet objectif à terme est atteint, IagosERI pourrait permettre à la communauté scientifique de capitaliser sur treize années d'investissement dans des programmes de recherche comme Mozaic et Caribic, conduisant à la mise au point d'un système d'observations global in situ par avion de nouvelle génération. Dans ce contexte, un ensemble instrumental très léger (environ $20 \mathrm{~kg}$ ) est en développement dans Iagos sous la coordination de l'université de Cambridge. Il pourrait permettre dans le futur une surveillance encore plus dense et à coût modéré de l'atmosphère chimique en donnant accès à encore plus de modèles d'avions commerciaux.

\section{Remerciements}

Le programme Mozaic n'a pu voir le jour que grâce aux énormes efforts déployés par Alain Marenco (Laboratoire d'aérologie). Les supports de la Commission européenne, d'Airbus et de l'Insu-CNRS ont été cruciaux pour la bonne marche des programmes. Nous remercions vivement les compagnies aériennes, Lufthansa, Austrian, Air France et l'ex-Sabena, pour le transport gratuit de l'instrumentation depuis 1994. Nos remerciements s'adressent aussi aux personnels de la Délégation régionale du CNRS (DR14) MidiPyrénées pour la gestion des contrats Mozaic et Iagos depuis leurs débuts. 


\section{Bibliographie}

: Baray J. L., S. Baldy, R. D. Diab et J.-P. Cammas, 2003 : Dynamical study of a tropical cut-off low over South Africa, and its impact on tropospheric ozone. Atmos. Environ., 37, 1475-1488.

: Barrie L., P. Borrell et J. Langen, 2004 : Atmospheric chemistry theme report: Integrated global atmospheric chemistry observations (IGACO), IGOS P-11.

http://ioc.unesco.org/igospartners/Atmosphere.htm

Borchi F. et A. Marenco, 2002 : Discrimination of air masses near the extratropical tropopause by multivariate analyses from Mozaic data. Atmos. Environ., 39, 1123-1135.

Borchi F., E. Oikonomou et A. Marenco, 2005 : Extratropical case study of stratosphere-troposphere exchange using multivariate analyses from Mozaic aircraft data. Atmos. Environ., 39, 6537-6549.

Bortz S. E., M. J. Prather, J.-P. Cammas, V. Thouret et H. Smit, 2006 : Ozone, water vapor, and temperature in the upper tropical troposphere: Variations over a

decade of Mozaic measurements. J. Geophys. Res., 111, D05305, DOl:10.1029/2005JD006512.

: Brasseur G. P., R. A. Cox, D Hauglustaine, I Isaksen, J. Lelieveld, D. H. Lister, R. Sausen, U. Schumann, A. Wahner et P. Wiesen, 1998 : European scientific - assessment of the atmospheric effects of aircraft emissions. Atmos. Environ., 32, 2329-2418.

- Bregman A., M. C. Krol, H. Teyssedre, W. A. Norton, A. Iwi, M. Chipperfield, G. Pitari, J. K. Sundet et J. Lelieveld, 2001 : Chemistry-transport model compari: son with ozone observations in the midlatitude lowermost stratosphere. J. Geophys. Res., 106 (D15): 17479-17496.

: Brenninkmeijer C. A. M., M. R. Manning, D. C. Lowe, G. Wallace, R. J. Sparks et A. Volz-Thomas, 1992 : Interhemispheric asymmetry in OH abundance inferred from measurements of atmospheric 14C0. Nature, 356, 50-52.

Brenninkmeijer C. A. M. et al., 2007 : Civil Aircraft for the regular investigation of the atmosphere based on an instrumented container: The new CaribicC system. Atmos. - Chem. Phys., 7, 4953-4976.

Brioude J., J.-P. Cammas et O. R. Cooper, 2006 : Stratosphere-troposphere exchange in a summertime extratropical low: analysis. Atmos. Chem. Phys., 6, $2337-2353$.

Brioude J., J.-P. Cammas, O. R. Cooper et P. Nédélec, 2008 : Characterization of the composition, structure ans seasonal variation of the mixing Mozaic measure: ments. J. Geophys. Res., 113, D00B01, D0I:10. 1029/2007JD009184.

- Cammas J.-P., S. Jakobi-Koali, K. Suhre, R. Rosset et A. Marenco, 1998 : Atlantic subtropical potential vorticity barrier as seen by Mozaic flights. J. Geophys. Res., : 103, 25681-25693.

: Cathala M. L., J. Pailleux et V.-H. Peuch, 2003 : Improving global simulations of UTLS ozone with assimilation of Mozaic data. Tellus, 55B, 1-10.

: Chai T., G. R. Carmichael, Y. Tang, A. Sandu, M. Hardesty, P. Pilewskie, S. Whitlow, E. V. Browell, M. A. Avery, P. Nédélec, J. T. Merrill, A. M. Thompson

- et $\mathbf{E}$. Williams, 2007 : Four-dimensional data assimilation experiments with international consortium for atmospheric research on transport and transformation ozone measurements. J. Geophys. Res., 112, D12S15.

- Chevalier A., F. Gheusi, R. Delmas, C. Ordóñez, C. Sarrat, R. Zbinden, V. Thouret, G. Athier et J.-M. Cousin, 2007 : Influence of altitude on ozone levels and varia-

: bility in the lower troposphere: a ground-based study for western Europe over the period 2001-2004. Atmos. Chem. Phys. , 7, 4311-4326.

: Cho J. Y. N., V. Thouret, R. E. Newell et A. Marenco, 2000 : Isentropic scaling analysis of ozone in the upper troposphere and lower stratosphere. J. Geophys. Res., - 106, 10 023-10 038.

- Cho J. Y. N. et E. Lindborg, 2001 : Horizontal velocity structure functions in the upper troposphere and lower stratosphere: 1. Observations. J. Geophys. Res., 106, 10223-10232.

- Clark H. L., M.-L. Cathala, H. Teyssedre, J.-P. Cammas et V.-H. Peuch, 2007 : Cross-tropopause fluxes of ozone using assimilation of Mozaic observations in a global : CTM. Tellus B 59, 39-49.

Clerbaux C. et al., 2007 : CO measurements from the ACE-FTS satellite instrument: data analysis and validation using ground-based, airborne and spaceborne observations. Atmos. Chem. Phys. Disc., 2007. 7, 15277-15340.

- Colette A., G. Ancellet et F. Borchi, 2005a : Impact of vertical transport processes on the tropospheric ozone layering above Europe. Part I: Study of air mass origin using : multivariate analysis, clustering and trajectories. Atmos. Env., 39, 5409-5422.

- Colette A. et G. Ancellet, 2005b : Impact of vertical transport processes on the tropospheric ozone layering above Europe. Part II: Climatological analysis of the past 30 years. Atmos. Environ, 39, 5423-5435.

Colette A. et G. Ancellet, 2006 : Variability of the tropospheric mixing and of streamer formation and their impact on the lifetime of observed ozone layers. Geophys. Res. - Lett., 33, L09808.

Cooper O. R., A. Stohl, S. Eckhardt, D. D Parrish, S. J. Oltmans, B. J. Johnson, P. Nédélec, F. J. Schmidlin, M. J. Newchurch, Y. Kondo et K. Kita, 2005 :

- A springtime comparison of tropospheric ozone and transport pathways on the east and west coasts of the United States. J. Geophys. Res, 110, D05S90.

- Cooper O. R. et al., 2006 : Large upper tropospheric ozone enhancements above midlatitude North America during summer: In situ evidence from the IONS and Mozaic : ozone measurement network. J. Geophys. Res., 111, D24S05.

Crowther R. A., K. S. Law, J. A. Pyle, S. Bekki et H. G. J. Smit, 2002 : Characterising the effect of large-scale model resolution upon calculated OH production using - Mozaic data. Geophys. Res. Lett., DOI 10.1029/2002GL014660.

- Dethof A, A. O'Neill, J. M. Slingo et al., 1999 : A mechanism for moistening the lower stratosphere involving the Asian summer monsoon. Quart. J. Roy. Meteor. Soc., : 125, 1079-1106.

- Diab R., A. Raghunandan, A. Thompson et V. Thouret, 2003 : Classification of tropospheric ozone profiles based on Mozaic aircraft data. Atmos. Chem. Phys. 3, 713-723.

Ding A. J., T. Wang, V. Thouret, J.-P. Cammas et P. Nédélec, 2007 : Tropospheric ozone climatology over Beijing: analysis of aircraft data from the Mozaic program. : Atmos. Chem. Phys. Discuss., 7, 9795-9828.

Emmons L. K., D. A. Hauglustaine, J-F. Muller, M. A. Caroll, G. P. Brasseur, D. Brunner, J. Staehelin, V. Thouret et A. Marenco, 2000 : Data composites of air- borne observations of tropospheric ozone and its precursors. J. Geophys. Res., 105, 20 497-20 538.

- Emmons L. K., G. G. Pfister, D. P. Edwards, J. C. Gille, G. Sachse, D. Blake, S. Wofsy, C. Gerbig, D. Matross et P. Nédélec, 2007 : Measurements of Pollution in : the Troposphere (MOPITT) validation exercises during summer 2004 field campaigns over North America. J. Geophys. Res., 112, D12S02, D0l:10.1029/2006JD007833.

- Gauss M., I. S. A. Isaksen, D. S. Lee et O. A. Søvde, 2006a : Impact of aircraft NOx emissions on the atmosphere - tradeoffs to reduce the impact. Atmos. Chem. :Phys., 6, 1529-1548.

Gheusi F. et al.,2007 : Ozone et pollution à grande échelle. Le réseau de surveillance PAES. La Météorologie, 8 e série, 58, 30-35.

: Gierens K., U. Schumann, H. G. J. Smit, M. Helten et G. Zängl, 1997 : Determination of humidity and temperature fluctuations based on Mozaic data and parametri: zation of persistent contrail coverage for general circulation models. Ann. Geophys., 15, 1057-1066.

- Gierens K., U. Schumann, M. Helten, H. G. J. Smit et A. Marenco, 1999 : A distribution law for relative humidity in the upper troposphere and lower stratosphere

- derived from three years of Mozaic measurements. Ann. Geophys., 17, 1218-1226. 
- Gierens K. et P. Spichtinger, 2000a : On the size distribution of ice-supersaturated regions in the upper troposphere and lowermost stratosphere. Ann. Geophys., 18, 499-504.

Gierens K., U. Schumann, M. Helten, H. Smit et P. H. Wang, 2000b : Ice-supersaturated regions and sub-visible cirrus in the northern midlatitude upper troposphere.

- J. Geophys. Res. 105, 22743-22754.

- Gouget H., G. Vaughan, A. Marenco et H. G. J. Smit., 2000 : Decay of a cut-off low and contribution to stratosphere-troposphere exchange. Ouart. J. Roy. Meteor. - Soc., 126, 1117-1142.

Helten M., H. Smit, W. Strater, D. Kley, P. Nédélec, M. Zoger et R. Busen, 1998 : Calibration and performance of automatic compact instrumentation for the measu- rement of relative humidity from passenger aircraft. J. Geophys. Res., 103, 25643-25652.

- Helten M., H. G. J. Smit, D. Kley, J. Ovarlez, H. Schlager, R. Baumann, U. Schumann, P. Nédélec et A. Marenco, 1999 : In-Flight Intercomparison of Mozaic and

- Polinat Water Vapor Measurements, J. Geophys. Res., 104, D21, 26087-26096.

- Heise S. et al., 2007 : Comparison of water vapour and temperature results from GPS radio occultations aboard Champ with Mozaic measurements. IEEE Trans. on - Geoscience and Remote Sensing, soumis.

- Isaksen I. S. A., R. Sausen, J. A. Pyle et al., 2003 : The EU project Tradeoff - Aircraft emissions: Contributions of various climate compounds to changes in composition - and radiative forcing - tradeoff to reduce atmospheric impact, Project Final report, Contract No. EVK2-CT-1999-0030 (available from the Department of Geosciences, University of Oslo, Oslo, Norway), 158

- Kalabokas P. D., A. Volz-Thomas, J. Brioude, V. Thouret, J.-P. Cammas et C. C. Repapis, 2007 : Vertical ozone measurements in the troposphere over the Eastern Mediterranean and comparison with Central Europe. Atmos. Chem. Phys. , 7, 3783-3790.

Kley D., H. G. J. Smit, S. Nawrath, Z. Luo, P. Nédélec et R. H. Johnson, 2007 : Tropical Atlantic convection as revealed by ozone and relative humidity measure- ments. J. Geophys. Res., sous presse.

Law K. S., P.-H. Plantevin, D. E. Shallcross, H. Rogers, C. Grouhel, V. Thouret, A. Marenco et J. A. Pyle, 1998 : Evaluation of modelled $0_{3}$ using Mozaic data. - J. Geophys. Res., 103, 25721-25740.

Law K. S., P. H. Plantevin, V. Thouret, A. Marenco, W. Asman, W. A. H. Lawrence, P. J. Crutzen, J. F. Muller, D. A. Hauglustaine et M. Kanakidou, 2000

- Comparison between global chemistry transport model results and measurements of ozone and water vapour by Airbus in-service aircraft (Mozaic) data. J. Geophys. Res., 105, 1503-1525

Li O., D. J. Jacob, J. A. Logan, I. Bey, R. M. Yantosca, H. Liu, R. V. Martin, A. M. Fiore, B. D. Field, B. N. Duncan et V. Thouret, 2001 : A tropospheric ozone - maximum over the Middle East. Geophys. Res. Lett., 28, 3235-3238.

- Lindborg E., 1999 : Can the atmospheric kinetic energy spectrum be explained by two-dimensional turbulence? J. Fluid. Mech., 388, 259-288.

- Lindborg E. et J. Y. N. Cho, 2000 : Determining the cascade of passive scalar variance in the lower stratosphere. Phys. Rev. Lett., 85, 5663-5666.

- Lindborg E. et J. Y. N. Cho, 2001 : Horizontal velocity structure functions in the upper troposphere and lower stratosphere: 2. Theoretical considerations, J. Geophys. Res., 106 (D10): 10233-10241.

Livesey N. J. et al., 2007 : Validation of EOS microwave limb sounder 03 and CO observations in the upper troposphere and lower stratosphere. J. Geophys. Res., sous presse.

- Luo Z., D. Kley, R. H. Johnson et H. Smit, 2007a : Ten years of measurements of tropical upper-tropospheric water vapor by Mozaic, Part I: climatology, variability, transport and relation to deep convection. J. Climate, 20, 418-435.

Kalabokas P. D., A. Volz-Thomas, J. Brioude, V. Thouret, J.-P. Cammas et C. C. Repapis, 2007 : Vertical ozone measurements in the troposphere over the Eastern - Mediterranean and comparison with Central Europe. Atmos. Chem. Phys., 7, 3783-3790.

Kley D., H. G. J. Smit, S. Nawrath, Z. Luo, P. Nédélec et R. H. Johnson, 2007 : Tropical Atlantic convection as revealed by ozone and relative humidity measurements. J. Geophys. Res., sous presse.

- Law K. S., P.-H. Plantevin, D. E. Shallcross, H. Rogers, C. Grouhel, V. Thouret, A. Marenco et J. A. Pyle, 1998 : Evaluation of modelled O ${ }_{3}$ using Mozaic data. - J. Geophys. Res., 103, 25721-25740.

Law K. S., P.-H. Plantevin, V. Thouret, A. Marenco, W. Asman, W. A. H. Lawrence, P. J. Crutzen, J. F. Muller, D. A. Hauglustaine et M. Kanakidou, 2000 - Comparison between global chemistry transport model results and measurements of ozone and water vapour by Airbus in-service aircraft (Mozaic) data. J. Geophys. Res., 105, 1503-1525

Li O., D. J. Jacob, J. A. Logan, I. Bey, R. M. Yantosca, H. Liu, R. V. Martin, A. M. Fiore, B. D. Field, B. N. Duncan et V. Thouret, 2001 : A tropospheric ozone - maximum over the Middle East. Geophys. Res. Lett., 28, 3235-3238.

- Lindborg E., 1999 : Can the atmospheric kinetic energy spectrum be explained by two-dimensional turbulence? J. Fluid. Mech., 388, 259-288.

- Lindborg E. et J. Y. N. Cho, 2000 : Determining the cascade of passive scalar variance in the lower stratosphere. Phys. Rev. Lett., 85, 5663-5666.

- Lindborg E. et J. Y. N. Cho, 2001 : Horizontal velocity structure functions in the upper troposphere and lower stratosphere: 2. Theoretical considerations, J. Geophys. Res., 106 (D10): 10233-10241.

Livesey N. J. et al., 2007 : Validation of EOS microwave limb sounder $\mathrm{O}_{3}$ and $\mathrm{CO}$ observations in the upper troposphere and lower stratosphere. J. Geophys. Res., sous presse.

- Luo Z., D. Kley, R. H. Johnson et H. Smit, 2007a : Ten years of measurements of tropical upper-tropospheric water vapor by Mozaic, Part I: climatology, variability, - transport and relation to deep convection. J. Climate, 20, 418-435.

- Luo Z., D. Kley, R. H. Johnson et H. Smit, 2007b : Ten years of measurements of tropical upper-tropospheric water vapor by Mozaic, Part II: Assessing the ECMWF Humidity Analysis. J. Clim. sous presse.

Marenco A., V. Thouret, P. Nédélec, H. Smit, M. Helten, D. Kley, F. Karcher, P. Simon, K. Law, J. Pyle, G. Poschmann, R. Von Wrede, C. Hume et T. Cook, - 1998 : Measurement of ozone and water vapour by Airbus in-service aircraft: the Mozaic airborne program, an overview. J. Geophys. Res., 103, $25631-25642$.

- Morgenstern 0. et A. Marenco, 2000 : Wintertime climatology of Mozaic ozone based on the potential vorticity and ozone analogy. J. Geophys. Res., 105, $15481-15493$.

- Morgenstern 0. et G. D. Carver, 2001 : Comparison of cross-tropopause transport and ozone in the upper troposphere and lower stratosphere region, J. Geophys. Res., 106 (D10), 10205-10222.

- Nastrom G. D., 1979 : Ozone in the upper troposphere from GASP measurements. J. Geophys. Res., 84, 6383-6388.

- Nédélec P., J.-P. Cammas, V. Thouret, G. Athier, J.-M. Cousin, C. Legrand, C. Abonnel, F. Lecœur, G. Cayez et C. Marizy, 2003 : An improved infra-red carbon - monoxide analyser for routine measurements aboard commercial Airbus aircraft: Technical validation and first scientific results of the Mozaic III Program. Atmos. Chem. - Phys. , 3, 1551-1564

- Nédélec P., V. Thouret, J. Brioude, B. Sauvage, J.-P. Cammas et A. Stohl, 2005 : Extreme CO concentrations in the upper troposphere over North-East Asia in June 2003 from the in-situ Mozaic aircraft data. Geophys. Res. Lett., 32, L14807.

- Nedoluha G. E., R. M. Bevilacque, K. W. Hoppel, J. D. Lumpe et H. G. J. Smit, 2002 : P0AM III measurements of water vapor in the upper troposphere and lower - most stratosphere. J. Geophys. Res., 107, D0I:10.1029/2001JD000793. 
- Newell R., V. Thouret, J. Cho, P. Stoller, A. Marenco et H. Smit, 1999 : Ubiquity of quasi-horizontal layers in the troposphere. Nature, 398, 316-319.

- Pätz H.-W., A. Volz-Thomas, M. I. Hegglin, D. Brunner, H. Fischer et U. Schmidt, 2006 : In-situ comparison of the NOy instruments flown in Mozaic and Spurt. - Atmos. Chem. Phys., 6, 2401-2410.

- Prados A. I., G. E. Nedoluha, R. M. Bevilacqua, D. R. Allen, K. W. Hoppelet et A. Marenco, 2003 : POAM III ozone in the upper troposphere and lowermost stratosphere: Seasonal variability and comparisons to aircraft observations. J. Geophys. Res., 108, D7, 10.1029/2002JD002819.

- Rogers H. L., 2005 : Scenic project: Scenario of aircraft emissions and impact studies on chemistry and climate: Final Report, EU Project EVK2-2001-00103.

- Sauvage B., V. Thouret, J.-P. Cammas, F. Gheusi, G. Athier et P. Nédélec, 2005 : Tropospheric ozone over Equatorial Africa: regional aspects from the Mozaic data. - Atmos. Chem. Phys., 5, 311-335.

- Sauvage B., V. Thouret, A. M. Thompson, J. Witte, J.-P. Cammas, P. Nédélec et G. Athier, 2006 : Enhanced view of the Tropical Atlantic ozone paradox and zonal - wave-one from the in-situ Mozaic and Shadoz data. J. Geophys. Res., 111, D1, D01301, D0I:10.1029/2005JD006241.

- Sauvage B., V. Thouret, J.-P. Cammas, J. Brioude, P. Nédélec et C. Mari, 2007a : Meridional ozone gradients in the African upper troposphere. Geophys. Res. Lett., : 34, L03817, DOI:10.1029/2006GL028542.

- Sauvage B., R. V. Martin, A. van Donkelaar et J. R. Ziemke, 2007b : Quantification of the factors controlling tropical tropospheric 0zone and the South Atlantic maxi: mum. J. Geophys. Res., 112, D11309, D0I:10.1029/2006JD008008.

: Sauvage B., F. Gheusi, V. Thouret, J.-P. Cammas, J. Duron, J. Escobar, C. Mari, P. Mascart et V. Pont, 2007c : Medium-range mid-tropospheric transport of ozone - and precursors over Africa: two numerical case-studies in dry and wet seasons, Atmos. Chem. Phys., sous presse.

: Scott R. K., J.-P. Cammas, P. Mascart et C. Stolle, 2001 : Stratospheric filamentation into the upper tropical troposphere. J. Geophys. Res., 106 (D11), $11835-11848$.

- Stohl A., P. James, C. Forster, N. Spichtinger, A. Marenco, V. Thouret et H. G. J. Smit, 2001 : An extension of measurements of ozone and water vapor by Airbus - in-service aircraft (Mozaic) ozone climatologies using trajectory statistics. J. Geophys. Res., 106, 27757-27768.

- Thouret V., A. Marenco, J. A. Logan, P. Nédélec et C. Grouhel, 1998a : Comparisons of ozone measurements from the Mozaic airborne program and the ozone soun- ding network at eight locations. J. Geophys. Res., 103, 25695-25720.

- Thouret V., A. Marenco, P. Nédélec et C. Grouhel, 1998b : Ozone climatologies at 9-12 km altitude as seen by the Mozaic airborne program between September 1994 - and August 1996. J. Geophys. Res., 103, 25653-25679.

: Thouret V., J. Y. N. Cho, R. E. Newell et al., 2000 : General characteristics of tropospheric trace constituent layers observed in the Mozaic program. J. Geophys. Res., 105 (D13), 17379-17392.

- Thouret V., J.-P. Cammas, B. Sauvage, G. Athier, R. Zbinden, P. Nédélec, P. Simon et F. Karcher, 2006 : Tropopause referenced ozone climatology and inter-annual - variability (1994-2003) from the Mozaic programme. Atmos. Chem. Phys., 6, 1033-1051.

- Tressol M., C. Ordóñez, R. Zbinden, V. Thouret, C. Mari, P. Nédélec, J.-P. Cammas, H. Smit, H.-W. Patz et A. Volz-Thomas, 2007 : Air pollution during the 2003 - European heat wave as seen by Mozaic airliners. Atmos. Chem. Phys. Discuss., October 2007.

- Volz A., D. H. Ehhalt et R. G. Derwent, 1981 : Seasonal and latitudinal variation of 14 CO and the tropospheric concentration of OH radicals. J. Geophys. Res., 86, 5163-5171.

: Volz-Thomas A., M. Berg, T. Heil, N. Houben, A. Lerner, W. Petrick, D. Raak et H.-W. Pätz, 2005 : Measurements of total odd nitrogen (NOy) aboard Mozaic in- service aircraft: instrument design, operation and performance. Atmos. Chem. Phys., 5, 583-595.

: Zbinden R. M., J.-P. Cammas, V. Thouret, P. Nédélec, F. Karcher et P. Simon, 2006 : Mid-latitude tropospheric ozone columns from the Mozaic program: climatology : and interannual variability. Atmos. Chem. Phys., 6, 1053-1073. 